\title{
The Quantum Strip: Liouville Theory for Open Strings
}

\author{
Eugène Cremmer and Jean-Loup Gervais ${ }^{\star}$ \\ Laboratoire de Physique Théorique de l'École Normale Supeŕieure, ${ }^{\star \star} 24$, rue Lhomond, \\ F-75231 Paris Cedex 05, France
}

Received March 15, 1991

\begin{abstract}
The quantum group structure of $2 \mathrm{D}$ gravity recently put forward by one of us (J.-L. G.) is used to study quantum gravity on the strip. The boundary conditions, previously studied by A. Neveu and this author become easy to implement when one introduces the universal family of chiral operators associated with $U_{q}(s l(2))$. A general formula for inverse powers of the metric-tensor operator is thereby derived. It contains a new universal matrix $A$, acting in representationspace, which obeys identities involving the $R$ matrix, the Clebsch-Gordon coefficients, and the co-products of $U_{q}(s l(2))$. The physical meaning of these identities is to ensure that these powers of the metric are local and closed by fusion.
\end{abstract}

\section{Introduction}

In the early days, Gervais and Neveu developed their operator-approach to Liouville theory by dealing mostly with open surfaces [1-6], and imposing classical boundary conditions derived from studies of the Weyl anomaly on surfaces with boundaries $[7,8]$. Since then, the algebraic approach initiated in refs. 3, 4 has made significant progress when its connection with quantum groups was recognized [9-13], but in recent times, 2D gravity with boundaries has received little attention apart from ref. 14. It is the aim of the present paper to go back to this problem. One will see that the quantum-group structure of refs. 9-13 leads to considerable simplifications and progress. Indeed, although Gervais and Neveu completely solved the classical problem on the strip [1,2], they could only derive the quantum expression for the simplest inverse power of the metric tensor [4], obtaining a rather complicated expression by imposing locality on the world-sheet. From the

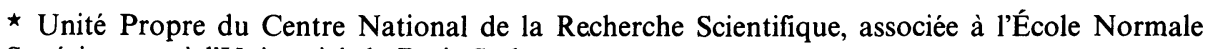
Supérieure et à l'Université de Paris-Sud

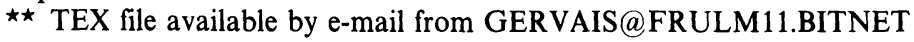


quantum-group viewpoint it corresponds to the simplest representation with spin $J=1 / 2$. In the present article we shall derive expressions for any negative powers of the metric corresponding to arbitrary representations of the underlying quantum group and check that they form a family of operators that are mutually local and closed by fusion.

At first (Sect. 2) we reconsider the case of spin 1/2 which was already solved in ref. 4. This allows to introduce notations and explain the background material contained in the earlier articles. The formula [4] for the corresponding power of the metric is re-expressed in terms of the spin- $1 / 2$ members of the universal family $[9,10]$ of chiral operators $\xi$, obtaining an expression which is remarkably simpler than that one of ref. 4 where the Block-wave basis was used. The operator-product expansion of the $\xi$ fields allows, next, to construct the powers of the metric corresponding to each of the two possible quantum parameters separately. This is the purpose of Sect. 3 where a universal form is derived that involves a matrix $A$ acting in the space of representations. In Sect. 4, it is shown that these operators are mutually local and closed by fusion. This is the consequence of quantum-group identities involving the $A$ matrix, together with the $R$ matrix, the Clebsch-Gordan coefficients, and the co-products of $U_{q}(s l(2))$. Finally, in Sect. 5, the above discussion is extended to the most general operator, that involves the two quantum-modifications together. A few concluding remarks are made in Sect. 6.

\section{The Case of Spin $1 / 2$}

At first we review the results of ref. 4 which will be our starting point. Simpler notations and normalisations will be introduced following ref. 10-13. We shall work on the strip $0 \leqq \sigma \leqq \pi,-\infty \leqq \tau \leqq+\infty$, using Minkowsky coordinates on the world-sheet. The boundary conditions is implemented by considering chiral fields inside the strip as well as outside, using an approach similar to the method of images in electromagnetism. Thus one introduces chiral fields on the covering space of the cylinder $0 \leqq \sigma \leqq 2 \pi,-\infty \leqq \tau \leqq+\infty$, which are the same for open and closed surfaces. We shall denote by $\gamma$ the Liouville coupling constant. Throughout this article we consider all operators at the same time $\tau$ which may be taken equal to zero without loss of generality and omitted. We are thus working on the unit circle $z=e^{i \sigma}$. First recall some basic formulae of refs. 10-13. For generic $\gamma$, there exist two equivalent free fields [3].

$$
\varphi_{j}(\sigma)=q_{0}^{(j)}+p_{0}^{(j)} \sigma+i \sum_{n \neq 0} e^{-i n \sigma} p_{n}^{(j)} / n, \quad j=1,2,
$$

such that (primes mean $\sigma$ derivatives)

$$
\begin{gathered}
{\left[\phi_{1}^{\prime}\left(\sigma_{1}\right), \phi_{1}^{\prime}\left(\sigma_{2}\right)\right]=\left[\phi_{2}^{\prime}\left(\sigma_{1}\right), \phi_{2}^{\prime}\left(\sigma_{2}\right)\right]=2 \pi i \delta^{\prime}\left(\sigma_{1}-\sigma_{2}\right), \quad p_{0}^{(1)}=-p_{0}^{(2)},} \\
N^{(1)}\left(\phi_{1}^{\prime}\right)^{2}+\phi_{1}^{\prime \prime} / \sqrt{\gamma}=N^{(2)}\left(\phi_{2}^{\prime}\right)^{2}+\phi_{2}^{\prime \prime} / \sqrt{\gamma}
\end{gathered}
$$

$N^{(1)}$ (respectively $N^{(2)}$ ) denote normal orderings with respect to the modes of $\phi_{1}$ (respectively $\phi_{2}$ ). Equation (2.3) defines the stress-energy tensor and the coupling constant $\gamma$ of the quantum theory. The former generates a representation of the Virasoro algebra with central charge $C=3+1 / \gamma$. 

or 2:

The chiral family is built up $[4-6,10]$ from the following operators with $j=1$

$$
\begin{aligned}
& \psi_{j}=d_{j} V_{j}, \quad V_{j}=N^{(j)}\left(e^{\sqrt{h / 2 \pi} \phi_{j}}\right), \quad \hat{\psi}_{j}=\hat{d}_{j} \hat{V}_{j}, \quad \hat{V}_{j}=N^{(j)}\left(e^{\sqrt{\hat{h} / 2 \pi} \phi_{J}}\right), \\
& h=\frac{\pi}{12}(C-13-\sqrt{(C-25)(C-1)}), \quad \hat{h}=\frac{\pi}{12}(C-13+\sqrt{(C-25)(C-1)}),
\end{aligned}
$$

where $d_{j}$ and $\hat{d}_{j}$ are normalization constants. The $V$ 's or the $\psi$ 's are determined as solutions of the equations

$$
\begin{aligned}
& -\psi_{j}^{\prime \prime}+\left(\frac{h}{\pi}\right)\left(\sum_{n<0} L_{n} e^{-i n \sigma}+\frac{L_{0}}{2}+\frac{C-1}{24}-\frac{h}{16 \pi}\right) \psi_{j}+\left(\frac{h}{\pi}\right) \psi_{j}\left(\sum_{n>0} L_{n} e^{-i n \sigma}+\frac{L_{0}}{2}\right)=0 \\
& -\hat{\psi}_{j}^{\prime \prime}+\left(\frac{\hat{h}}{\pi}\right)\left(\sum_{n<0} L_{n} e^{-i n \sigma}+\frac{L_{0}}{2}+\frac{C-1}{24}-\frac{\hat{h}}{16 \pi}\right) \hat{\psi}_{j}+\left(\frac{\hat{h}}{\pi}\right) \hat{\psi}_{j}\left(\sum_{n>0} L_{n} e^{-i n \sigma}+\frac{L_{0}}{2}\right)=0
\end{aligned}
$$

These are operator Schrödinger-equations equivalent to the decoupling of Virasoro null-vectors [4-6]. Since there are two possible quantum-modifications $h$ and $\hat{h}$, there are four solutions ${ }^{1}$. By operator-product expansions, $\psi_{j}, j=1,2$, and $\hat{\psi}_{j}$, $j=1,2$, generate two infinite families of chiral fields which are denoted $\psi_{m}^{(J)}$, $-J \leqq m \leqq J$, and $\hat{\psi}_{\hat{m}}^{(\hat{J})},-\hat{J} \leqq \hat{m} \leqq \hat{J}$, respectively; with $\psi_{-1 / 2}^{(1 / 2)}=\psi_{1}, \psi_{1 / 2}^{(1 / 2)}=\psi_{2}$, and $\hat{\psi}_{-1 / 2}^{(1 / 2)}=\hat{\psi}_{1}, \hat{\psi}_{1 / 2}^{(1 / 2)}=\hat{\psi}_{2}$. An easy computation shows that the standard screening charges are equal to $-\alpha_{ \pm}$where

$$
\alpha_{-}=\sqrt{2 h / \pi}, \quad \alpha_{+}=\sqrt{2 \hat{h} / \pi}
$$

$\psi_{m}^{(J)}, \quad \hat{\psi}_{m}^{(\hat{J})}$, are of the type $(1,2 J+1)$ and $(2 \hat{J}+1,1)$, respectively, in the BPZ classification. For the zero-modes, it is simpler [10] to define the rescaled variables

$$
\varpi=i p_{0}^{(1)} \sqrt{2 \pi / h} ; \quad \hat{\varpi}=i p_{0}^{(1)} \sqrt{2 \pi / \hat{h}} ; \quad \hat{\varpi}=\varpi h / \pi ; \quad \varpi=\hat{\varpi} \hat{h} / \pi .
$$

The commutation relations (2.2) are to be supplemented by the zero-mode ones:

$$
\left[q_{0}^{(1)}, p_{0}^{(1)}\right]=\left[q_{0}^{(2)}, p_{0}^{(2)}\right]=i .
$$

The fields $\psi$ and $\hat{\psi}$ shift the quasi momentum $p_{0}^{(1)}=-p_{0}^{(2)}$ by a fixed amount. For an arbitrary $c$-number function $f$ one has

$$
\psi_{m}^{(J)} f(\varpi)=f(\varpi+2 m) \psi_{m}^{(J)}, \quad \hat{\psi}_{m}^{(\hat{J})} f(\varpi)=f(\varpi+2 \hat{m} \pi / h) \hat{\psi}_{m}^{(\hat{J})}
$$

For the time being, we shall consider the hatted and unhatted fields separately. Since they are quite similar, we shall only discuss one family explicitly and deal with the unhatted fields. Any expression we write down has its hatted counterpart. Making use of the notations just recalled, the local operator of ref. 4 (formula (3.1) of that article) reads ${ }^{2}$ (we denote $2 \pi-\sigma$ by $\bar{\sigma}$ )

$$
\begin{aligned}
e^{-\alpha-\Phi / 2}= & \sqrt{\frac{h}{4 \pi}}\left[b(\varpi) V_{1}(\sigma) V_{1}(\bar{\sigma})+a(\varpi) V_{1}(\sigma) V_{2}(\bar{\sigma})\right. \\
& \left.-d(\varpi) V_{2}(\sigma) V_{1}(\bar{\sigma})-c(\varpi) V_{2}(\sigma) V_{2}(\bar{\sigma})\right] .
\end{aligned}
$$

\footnotetext{
1 The operators $V_{1}$ and $V_{2}$ are equal to the operators $\psi_{1}$ and $\psi_{2}$ of ref. 4 , respectively.

${ }^{2}$ Up to a few misprints
} 
$a, b, c, d$ which are only functions of $\varpi$ were determined in ref. 4 by imposing that

$$
e^{-\alpha-\Phi(\sigma) / 2} e^{-\alpha-\Phi\left(\sigma^{\prime}\right) / 2}=e^{-\alpha-\Phi\left(\sigma^{\prime}\right) / 2} e^{-\alpha-\Phi(\sigma) / 2}
$$

as is required by locality. Their expression will be given below. The relations between $V$ and $\psi$ fields explicitly $\operatorname{read}^{3}\left(\psi_{ \pm 1 / 2}(1 / 2)\right.$ are simply written as $\left.\psi_{ \pm 1 / 2}\right)$

$$
\begin{aligned}
\psi_{-11 / 2}(\sigma) & =\sqrt{\Gamma_{0}(-\varpi h / \pi) \Gamma_{0}((\varpi-1) h / \pi)} V_{1}(\sigma), \\
\psi_{11 / 2}(\sigma) & =\sqrt{\Gamma_{0}(-(\varpi+1) h / \pi) \Gamma_{0}(\varpi h / \pi)} V_{2}(\sigma) .
\end{aligned}
$$

For our purpose, it is better to write the expression in terms of the $\psi$ fields as

$$
\begin{aligned}
e^{-\alpha-\Phi / 2}= & \frac{1}{\pi} \sqrt{\frac{h}{4 \pi}}\left[\psi_{-1 / 2}(\sigma) \beta(\varpi) \psi_{-1 / 2}(\bar{\sigma})+\psi_{-1 / 2}(\sigma) \alpha(\varpi) \psi_{1 / 2}(\bar{\sigma})\right. \\
& \left.-\psi_{1 / 2}(\sigma) \delta(\varpi) \psi_{-1 / 2}(\bar{\sigma})-\psi_{1 / 2}(\sigma) \gamma(\varpi) \psi_{1 / 2}(\bar{\sigma})\right] .
\end{aligned}
$$

In this way, the $\alpha, \ldots, \gamma$ have a simple relationship with the rescaled quantities, denoted by $\tilde{a}, \ldots, \tilde{d}$ of ref. 4 . On the one hand, one has

$$
\alpha(\varpi)=\tilde{a}(\varpi+1) e^{-i h(\varpi+1) / 2}, \quad \delta(\varpi)=\tilde{d}(\varpi-1) e^{i h(\varpi-1) / 2},
$$

and this gives

$$
\alpha(\varpi)=e^{-i h / 4}\left(\rho_{1}+\rho_{2} e^{-i h \varpi}\right), \quad \delta(\varpi)=-e^{-i h / 4}\left(\rho_{1}+\rho_{2} e^{i h \varpi}\right) .
$$

The two parameters $\rho_{1}$ and $\rho_{2}$ are the quantum-analogues of the classical parameters relevant for the boundary conditions at $\sigma=0$ and $\sigma=\pi$ respectively. At the quantum level, they appeared in ref. 4 as integration constants in solving (2.12). We shall come back to this point below. On the other hand, condition (2.12) only determines the product $\tilde{b}(\varpi+1) \tilde{c}(\varpi-1)$. One has

$$
\beta(\varpi) \gamma(\varpi)=\sin [h(\varpi+1)] \sin (h \varpi) \tilde{b}(\varpi+1) \tilde{c}(\varpi-1) .
$$

Equation (3.8) of ref. 4 becomes

$$
\beta(\varpi) \gamma(\varpi)-\alpha(\varpi) \delta(\varpi)=\sin ^{2}(h \varpi) e^{-i h / 2},
$$

and the product $\beta \gamma$ is given by

$$
\beta(\varpi) \gamma(\varpi)=e^{-i h / 2}\left[\sin ^{2}(h \varpi)-\rho_{1}^{2}-\rho_{2}^{2}-2 \rho_{1} \rho_{2} \cos (h \varpi)\right] .
$$

The $\psi$ fields have simple shift-properties for the zero-mode $\varpi$; they are closed under O.P.E. and braiding, and obey a quantum-group symmetry of the $U_{q}(s l(2))$-type. However, the fusion coefficients and $R$-matrix elements depend upon $\varpi$ and thus do not commute with the $\psi$ 's. Their explicit form is unusual, therefore. One may exhibit the standard $U_{q}(s l(2))$-quantum-group structure by changing basis to a new family of operators denoted by $\xi$. Following ref. 10 we introduce

$$
\left.\xi_{M}^{(J)}(\sigma):=\sum_{-J \leqq m \leqq J} \mid J, \varpi\right)_{M}^{m} \psi_{m}^{(J)}(\sigma), \quad-J \leqq M \leqq J
$$

\footnotetext{
${ }^{3}$ Since we want to adopt the same notations as in ref. 12, the ordinary gamma functions (not $q$-deformed) are characterised by an index zero (they correspond to a vanishing parameter $h$ ). The $q$-deformed gamma function with parameter $h$ is denoted by $\Gamma(z)$
} 


$$
\begin{aligned}
\mid J, \varpi)_{M}^{m}= & \sqrt{\left(\begin{array}{c}
2 J \\
J+M
\end{array}\right) e^{i h m / 2}} \\
& \sum_{(J-M+m-t / 2) \text { integer }} e^{i h t(\varpi+m)}\left(\begin{array}{c}
J-M \\
(J-M+m-t) / 2
\end{array}\right)\left(\begin{array}{c}
J+M \\
(J+M+m+t) / 2
\end{array}\right), \\
& \left(\begin{array}{l}
P \\
Q
\end{array}\right) \equiv \frac{\lfloor P\rfloor !}{\lfloor Q\rfloor !\lfloor P-Q\rfloor !}\lfloor n\rfloor ! \equiv \prod_{r=1}^{n}\lfloor r\rfloor \quad\lfloor r\rfloor \equiv \frac{\sin (h r)}{\sin h} .
\end{aligned}
$$

The last equation introduces $q$-deformed factorials and binomial coefficients. The fields $\xi_{M}^{(J)}$ for $-J \leqq M \leqq J$, span a representation of spin $J$ of $U_{q}(\operatorname{sl}(2))$. We shall work for generic central charge $C$, so that $e^{i h}$ and $e^{i \hat{h}}$ are not roots of unity; and the representations are simple deformations of the $s l(2)$-case.

In this article, we are primarily aiming at the weak coupling regime where $h$, $\hat{h}$, and eigenvalue-spectrum of $\varpi$ are real. In this case, it was shown in ref. 10 , that the $\xi$-fields satisfy the hermiticity relation

$$
\xi_{M}^{(J)}(\sigma)^{\dagger} \div \xi_{M}^{(J)}(\sigma)
$$

Next we re-express formula (2.11) in terms of the fields $\xi_{ \pm 1 / 2}^{(1 / 2)}$ which we simply write $\xi_{ \pm 1 / 2}$. Formula (2.20) gives

$$
\xi_{ \pm 1 / 2}=e^{i h(\pi \pm 1) / 2} \psi_{ \pm 1 / 2}+e^{-i h \pi / 2} \psi_{\mp 1 / 2} .
$$

By inverting this relation one finds

$$
\psi_{ \pm 1 / 2}=\frac{1}{2 i \sin (h \varpi)}\left[e^{i h(\varpi \mp 1) / 2} \xi_{ \pm 1 / 2}-e^{-i h \varpi / 2} \xi_{\mp 1 / 2}\right],
$$

or, equivalently ${ }^{4}$,

$$
\psi_{ \pm 1 / 2}=\left[\xi_{ \pm 1 / 2} e^{i h(\varpi) / 2}-\xi_{\mp 1 / 2} e^{-i h(\varpi \pm 1) / 2}\right] \frac{1}{2 i \sin (h \varpi)} .
$$

Substitute into Eq. (2.13). One gets

$$
e^{-\alpha-\Phi / 2}=\sqrt{\frac{h}{16 \pi^{3}}} \sum_{M, N=\mp 1 / 2} \xi_{M}(\sigma) A_{M, N} \xi_{N}(\bar{\sigma}),
$$

where

$$
\begin{aligned}
A_{-1 / 2,-1 / 2} & =\left[-\beta(\varpi) e^{i h(\varpi+1 / 2)}+\gamma(\varpi) e^{-i h(\varpi+1 / 2)}+\alpha(\varpi)-\delta(\varpi)\right] / 2 \sin ^{2} h \varpi, \\
A_{1 / 2,1 / 2} & =\left[-\beta(\varpi) e^{i h(-\varpi+1 / 2)}+\gamma(\varpi) e^{i h(\varpi-1 / 2)}+\alpha(\varpi)-\delta(\varpi)\right] / 2 \sin ^{2} h \varpi, \\
A_{-1 / 2,1 / 2} & =\left[\beta(\varpi)-\gamma(\varpi) e^{-i h}-\alpha(\varpi) e^{i h(\varpi-1 / 2)}+\delta(\varpi) e^{-i h(\varpi+1 / 2)}\right] / 2 \sin ^{2} h \varpi, \\
A_{1 / 2,-1 / 2} & =\left[\beta(\varpi) e^{i h}-\gamma(\varpi)-\alpha(\varpi) e^{-i h(\varpi-1 / 2)}+\delta(\varpi) e^{-i h(\varpi+1 / 2)}\right] / 2 \sin ^{2} h \varpi .
\end{aligned}
$$

So far the coefficients $\alpha, \ldots, \delta$ are not completely determined. The last four equations

${ }^{4}$ Note that the braiding between $\varpi$ and the fields $\xi$ is non-trivial 
imply

$$
\begin{aligned}
A_{-1 / 2,-1 / 2}+A_{1 / 2,1 / 2}+e^{i h / 2} \cos (h \varpi) A_{-1 / 2,1 / 2} & =2 \rho_{1} e^{-i h / 4}, \\
e^{i h} A_{-1 / 2,1 / 2}-A_{1 / 2,-1 / 2} & =-2 \rho_{2} e^{i h / 4} .
\end{aligned}
$$

In the $\xi$-basis, it is natural to choose, if possible, $A_{M, N}$ independent of $\varpi$. Equations (2.32), (2.33) then imply

$$
\begin{aligned}
A_{-1 / 2,1 / 2}=0, \quad A_{1 / 2,-1 / 2} & =2 \rho_{2} e^{i h / 4}, \\
A_{-1 / 2,-1 / 2}+A_{1 / 2,1 / 2} & =2 \rho_{1} e^{-i h / 4} .
\end{aligned}
$$

This completely determines the coefficients $A_{M, N}$ as well as $\beta(\varpi)$ and $\gamma(\varpi)$. Indeed Eqs. (2.16), (2.30) give

$$
\beta e^{i h / 2}-\gamma e^{-i h / 2}=2 e^{-i h / 4}\left(\rho_{1} \cos (h \varpi)+\rho_{2}\right),
$$

and, combining with (2.19)

$$
\begin{aligned}
& \beta(\varpi)=e^{-3 i h / 4}\left(\rho_{1} \cos (h \varpi)+\sqrt{1-\rho_{1}^{2}} \sin (h \varpi)+\rho_{2}\right), \\
& \gamma(\varpi)=-e^{i h / 4}\left(\rho_{1} \cos (h \varpi)-\sqrt{1-\rho_{1}^{2}} \sin (h \varpi)+\rho_{2}\right) .
\end{aligned}
$$

Substitute into the expressions Eqs. (2.14)-(2.31) for $e^{-\alpha-\Phi / 2}$. One gets

$$
\begin{aligned}
e^{-\alpha-\Phi / 2}= & \sqrt{\frac{h}{16 \pi^{3}}}\left\{-i e^{-i h / 4} e^{i \Omega_{1}} \xi_{-1 / 2}(\sigma) \xi_{-1 / 2}(\bar{\sigma})\right. \\
& \left.+i e^{-i h / 4} e^{-i \Omega_{1}} \xi_{1 / 2}(\sigma) \xi_{1 / 2}(\bar{\sigma})+2 \rho_{2} e^{i h / 4} \xi_{1 / 2}(\sigma) \xi_{-1 / 2}(\bar{\sigma})\right\}
\end{aligned}
$$

where we have let $\rho_{1}=\sin \Omega_{1}$. On the original expression Eq. (2.14), one sees that the field $\exp \left(-\alpha_{-} \Phi / 2\right)$ is hermitian, as shown in ref. 4, for $\rho_{1}$ and $\rho_{2}$ real, and $\gamma=-\beta^{*} e^{-i h / 2}$. This last condition is compatible with. Eqs. $(2.37,2.38)$ only for $\rho_{1}^{2} \geqq 1$. Indeed, according to (2.23), Eq. (2.39) violates hermiticity for real $\rho_{1}$ and $\rho_{2}$ except for $\Omega_{1}+\pi / 2$ pure imaginary. What happens is that, unless this last condition is satisfied, it is not possible to choose the coefficients $A_{M, N}$ to be independent of $\varpi$, and preserve hermiticity. We shall restrict ourselves to the case $\rho_{1}=1$ for simplicity Our starting point will thus be the formula

$$
e^{-\alpha-\Phi / 2}=\frac{1}{2 \pi} \sqrt{\frac{h}{2 \pi}}\left\{e^{-i h / 4}\left[\xi_{-1 / 2}(\sigma) \xi_{-1 / 2}(\bar{\sigma})+\xi_{1 / 2}(\sigma) \xi_{1 / 2}(\bar{\sigma})\right]+\kappa e^{i h / 4} \xi_{1 / 2}(\sigma) \xi_{-1 / 2}(\bar{\sigma})\right\}
$$

where $\kappa=2 \rho_{2}$. This expression has a triangular form which is remarkably simple.

The present discussion is not symmetric between $\rho_{1}$ and $\rho_{2}$ except if they are both equal to one. This is due to the fact the original expression Eq. (2.14) treats the two boundaries $\sigma=0$ and $\sigma=\pi$ differently. Indeed at the latter boundary, $\sigma$ and $\bar{\sigma}$ coincide, while they differ by $2 \pi$ in the former. One may exchange the role of the boundaries using the monodromy properties of the chiral fields. The $\psi$-fields satisfy

$$
\psi_{ \pm 1 / 2}(\sigma+2 \pi)=e^{ \pm i h \pi} e^{i h / 2} \psi_{ \pm 1 / 2}(\sigma) .
$$

For later use, we note that this last equation also determines the monodromy 
properties of the $\xi$ fields. Using $(2.24-2.26)$, one obrains:

$$
\begin{gathered}
\xi_{-1 / 2}(\sigma+2 \pi)=e^{i h} \xi_{1 / 2}(\sigma), \\
\xi_{1 / 2}(\sigma+2 \pi)=2 e^{i h / 2} \cos (h \varpi) \xi_{1 / 2}(\sigma)-\xi_{-1 / 2}(\sigma) .
\end{gathered}
$$

In the $\xi$-basis, the monodromy matrix is not diagonal, but only triangular. It depends on $\varpi$ and thus does not commute with the $\xi$ fields. For instance, one also has

$$
\xi_{1 / 2}(\sigma+2 \pi)=2 e^{-i h / 2} \xi_{1 / 2}(\sigma) \cos (h \varpi)-e^{-i 2 h} \xi_{-1 / 2}(\sigma) .
$$

Coming back to our discussion, let us note that Eq. (2.14) is equivalent to

$$
\begin{aligned}
e^{-\alpha-\Phi / 2}= & \frac{e^{i h / 2}}{\pi} \sqrt{\frac{h}{4 \pi}}\left[\psi_{-1 / 2}(\sigma) \bar{\beta}(\varpi) \psi_{-1 / 2}(-\sigma)+\psi_{-1 / 2}(\sigma) \bar{\alpha}(\varpi) \psi_{1 / 2}(-\sigma)\right. \\
& \left.-\psi_{1 / 2}(\sigma) \bar{\delta}(\varpi) \psi_{-1 / 2}(-\sigma)-\psi_{1 / 2}(\sigma) \bar{\gamma}(\varpi) \psi_{1 / 2}(-\sigma)\right],
\end{aligned}
$$

where

$$
\bar{\beta}=\beta e^{-i h \omega}, \quad \bar{\delta}=\delta e^{-i h \omega}, \quad \bar{\alpha}=\alpha e^{i h \pi}, \quad \bar{\gamma}=\gamma e^{i h \pi} .
$$

In the above discussion, it is easy to see that, using the new coefficients is equivalent to exchanging $\rho_{1}$ with $\rho_{2}$ and $\alpha$ with $-\delta$. Thus the form (2.45) allows us to deal with $\rho_{2}=1$ and $\rho_{1}$ real. The expression is triangular in the opposite way. We shall leave out this case which is similar to $(2.40)$.

Let us now discuss the physical meaning of the $\rho$ parameters. At the level of classical mechanics they were introduced in $[1,2]$ as characterising the boundary conditions at $\sigma=0$ and $\sigma=\pi$, and this is our next topic. It will be pedagogical to use the classical limit of the formulae just discussed, since they are much simpler than the ones of refs. 1, 2 and will be directly related to the quantum boundaryconditions to be considered later on. In the limit $\gamma \rightarrow 0, h \sim 2 \pi \gamma$ and $\alpha_{-} \sim 2 \sqrt{\gamma}$. The classical Liouville field of refs. 1,2 is $\tilde{\Phi} \sim \alpha_{-} \Phi+2 \ln \alpha_{-}$. The boundary conditions of refs. 1,2 are

$$
\begin{aligned}
& \partial e^{-\tilde{\Phi} / 2} /\left.\partial \sigma\right|_{\sigma=0}=\rho_{1} / \sqrt{2}, \\
& \partial e^{-\tilde{\Phi} / 2} /\left.\partial \sigma\right|_{\sigma=\pi}=-\rho_{2} / \sqrt{2},
\end{aligned}
$$

and we are going to rederivative them (with $\rho_{1}=1$ ) from the classical limit of Eq. (2.40). The limit $\gamma \rightarrow 0$ is taken with $\varpi \rightarrow \infty$ so that $h \varpi$ has a finite limit which we denote with the same symbols. For instance, Eqs. (2.24-2.26) become, respectively,

$$
\begin{gathered}
\xi_{ \pm 1 / 2}=e^{i h \varpi / 2} \psi_{ \pm 1 / 2}+e^{-i h \varpi / 2} \psi_{\mp 1 / 2}, \\
\psi_{ \pm 1 / 2}=\left[e^{i h \varpi / 2} \xi_{ \pm 1 / 2}-e^{-i h \pi / 2} \xi_{\mp 1 / 2}\right] /(2 i \sin (h \varpi)),
\end{gathered}
$$

where now $h \varpi=2 \pi \gamma \varpi$. Clearly $h \varpi$ must have a finite limit since otherwise the classical limit would be singular, as shown for instance in (2.50). Equation (2.40) becomes

$$
e^{-\tilde{\Phi} / 2}=\frac{1}{4 \pi \sqrt{2}}\left\{\xi_{-1 / 2}(\sigma) \xi_{-1 / 2}(\bar{\sigma})+\xi_{1 / 2}(\sigma) \xi_{1 / 2}(\bar{\sigma})+\kappa \xi_{1 / 2}(\sigma) \xi_{-1 / 2}(\bar{\sigma})\right\}
$$


The boundary condition involves $\partial e^{-\tilde{\Phi} / 2} / \partial \sigma$ at $\sigma=0$ and $\sigma=\pi$, which are computed from the Wronskian of the $\xi$ classical functions. The latter is derived in Appendix A. One has

$$
W\left[\xi_{1 / 2}^{(1 / 2)}, \xi_{-1 / 2}^{(1 / 2)}\right] \equiv \xi_{1 / 2}^{(1 / 2)}(\sigma) \frac{d}{d \sigma} \xi_{-1 / 2}^{(1 / 2)}(\sigma)-\xi_{-1 / 2}^{(1 / 2)}(\sigma) \frac{d}{d \sigma} \xi_{1 / 2}^{(1 / 2)}(\sigma)=2 \pi .
$$

It is independent of $\sigma$ since $\xi_{ \pm 1 / 2}^{(1 / 2)}$ are solutions of the same Schrödinger equation which is the classical limit of (2.6). Consider next the boundary $\sigma=\pi$. One immediately obtains

$$
\partial e^{-\tilde{\boldsymbol{\Phi}} / 2} /\left.\partial \sigma\right|_{\sigma=\pi}=-\frac{\kappa}{4 \pi \sqrt{2}} W\left[\xi_{1 / 2}^{(1 / 2)}, \xi_{-1 / 2}^{(1 / 2)}\right]=-\frac{1}{\sqrt{2}} \rho_{2}
$$

in agreement with refs. 1, 2 (Eq. (2.44)). Consider, next the boundary $\sigma=0$. The method is similar, but we have to first transform (2.51) using the monodromy properties of the $\xi$ fields, described by the classical limits of Eqs. $(2.42-2.44)$. One gets

$$
\begin{aligned}
e^{-\tilde{\Phi} / 2}= & \frac{1}{4 \pi \sqrt{2}}\left\{\xi_{1 / 2}(\sigma) \xi_{-1 / 2}(-\sigma)-\xi_{-1 / 2}(\sigma) \xi_{1 / 2}(-\sigma)\right. \\
& \left.+\xi_{1 / 2}(\sigma)(2 \cos (h \varpi)+2 \kappa) \xi_{1 / 2}(-\sigma)\right\}
\end{aligned}
$$

and the same calculation as above gives

$$
\partial e^{-\tilde{\Phi} / 2} /\left.\partial \sigma\right|_{\sigma=0}=1 / \sqrt{2}
$$

in agreement with refs. 10, 11 (Eq. (2.47)) with $\rho_{1}=1$.

Finally we turn to the quantum equivalent of the boundary conditions (2.48) (we leave out the boundary $\sigma=0$ which is treated in the same way). The quantumequivalent of the Wronskian is discussed in Appendix A. It is replaced by the following condition on the short-distance operator-product expansion. For $\sigma \rightarrow \sigma^{\prime}$, one has, according to (A.24),

$$
\xi_{-1 / 2}^{(1 / 2)}(\sigma) \xi_{1 / 2}^{(1 / 2)}\left(\sigma^{\prime}\right)-e^{i h} \xi_{1 / 2}^{(1 / 2)}(\sigma) \xi_{-1 / 2}^{(1 / 2)}\left(\sigma^{\prime}\right) \sim\left(d\left(\sigma-\sigma^{\prime}\right)\right)^{1+3 h / 2 \pi}\left(-2 i \pi e^{i h / 2}\right) \frac{\Gamma_{0}(1+h \pi)}{\Gamma_{0}(2+2 h \pi)} .
$$

The left-hand side is rewritten by using the braiding relation

$$
\xi_{-1 / 2}^{(1 / 2)}(\sigma) \xi_{1 / 2}^{(1 / 2)}\left(\sigma^{\prime}\right)=e^{i h / 2} \xi_{1 / 2}^{(1 / 2)}\left(\sigma^{\prime}\right) \xi_{-1 / 2}^{(1 / 2)}(\sigma) .
$$

Starting from Eq. (2.40), a straightforward computation shows that, for $\varepsilon \rightarrow 0$, one has

$$
\frac{\left.e^{i h / 4} e^{-\alpha-\Phi / 2}\right|_{\sigma=\pi+\varepsilon}-\left.e^{-i h / 4} e^{-\alpha-\Phi / 2}\right|_{\sigma=\pi-\varepsilon}}{i(2 i \varepsilon)^{1+3 h / 2 \pi}} \sim \rho_{2} \sqrt{\frac{h}{\pi}} \frac{\Gamma_{0}(1+h / \pi)}{\Gamma_{0}(2+2 h / \pi)} .
$$

The left-hand side is the quantum equivalent of the $\sigma$ derivative at $\sigma=\pi$. It is of course modified to take account of the quantum-corrections to the Taylor expansion. 


\section{Recursive Construction for Arbitrary $J$}

In this section, we construct the operators $e^{-\alpha-J \Phi}$ for arbitrary positive integer $2 J$ by using the operator-product expansion of the $\xi$ fields. The normalisations will be chosen so that the leading-order fusion-rule is given by

$$
e^{-\alpha-J \Phi(\sigma)} e^{-\alpha-J^{\prime} \Phi\left(\sigma^{\prime}\right)} \sim\left(d\left(\sigma-\sigma^{\prime}\right)\right)^{-2 h J J^{\prime} / \pi} e^{-\alpha_{-}\left(J+J^{\prime}\right) \Phi(\sigma)},
$$

where $d\left(\sigma-\sigma^{\prime}\right)=1-e^{-i\left(\sigma-\sigma^{\prime}\right)}$. Some general information concerning our conventions for primary fields on the unit circle is recalled at the end of the appendix, following refs. $10,11,13$. We shall omit the divergent factor $\left(d\left(\sigma-\sigma^{\prime}\right)\right)^{-2 h J J^{\prime} / \pi}$ in the following. Motivated by Eq. (2.27), we shall start with the ansatz

$$
e^{-\alpha-J \Phi(\sigma)}=\left(\frac{h}{16 \pi^{3}}\right)^{J} \sum_{M, N} A_{M, N}^{(J)} \xi_{M}^{(J)}(\sigma) \xi_{N}^{(J)}(\bar{\sigma}),
$$

where $A_{M, N}^{(J)}$ are constants to be determined, and

$$
A_{1 / 2,1 / 2}^{(1 / 2)}=A_{-1 / 2,-1 / 2}^{(1 / 2)}=e^{-i h / 4}, \quad A_{1 / 2,-1 / 2}^{(1 / 2)}=\kappa e^{i h / 4}, \quad A_{-1 / 2,1 / 2}^{(1 / 2)}=0 .
$$

In order to proceed, we recall two more relevant results of ref. 10:

I. To leading order, the fusion of two $\xi$ fields is given by

where

$$
\xi_{M}^{(J)}(\sigma) \xi_{M^{\prime}}^{\left(J^{\prime}\right)}\left(\sigma^{\prime}\right) \sim \lambda\left(J, M ; J^{\prime}, M^{\prime}\right) \xi_{M+M^{\prime}}^{\left(J+J^{\prime}\right)}(\sigma),
$$

$$
\lambda\left(J, M ; J^{\prime}, M^{\prime}\right)=\frac{\left(\begin{array}{c}
2 J \\
J+M
\end{array}\right)\left(\begin{array}{c}
2 J^{\prime} \\
J^{\prime}+M^{\prime}
\end{array}\right)}{\left(\begin{array}{c}
2 J+2 J^{\prime} \\
J+J^{\prime}+M+M^{\prime}
\end{array}\right)} e^{i h\left(M^{\prime} J-M J^{\prime}\right)} .
$$

II. The braiding properties are

1) For $\pi>\sigma>\sigma^{\prime}>0$, the $\xi$ fields obey the exchange algebra

$$
\begin{gathered}
\xi_{M}^{(J)}(\sigma) \xi_{M^{\prime}}^{\left(J^{\prime}\right)}\left(\sigma^{\prime}\right)=\sum_{-J \leqq N \leqq J ;-J^{\prime} \leqq N^{\prime} \leqq J^{\prime}}\left(J, J^{\prime}\right)_{M M^{\prime}}^{N^{\prime} N} \xi_{N^{\prime}}^{\left(J^{\prime}\right)}\left(\sigma^{\prime}\right) \xi_{N}^{(J)}(\sigma), \\
\left(J, J^{\prime}\right)_{M M^{\prime}}^{N^{\prime} N}=\left(\left\langleJ, M\left|\otimes\left\langle J^{\prime}, M^{\prime}\right|\right) \mathbf{R}\left(|J, N\rangle \otimes\left|J^{\prime}, N^{\prime}\right\rangle\right),\right.\right. \\
\mathbf{R}=e^{\left(-2 i h J_{3} \otimes J_{3}\right)}\left(1+\sum_{n=1}^{\infty} \frac{\left(1-e^{2 i h}\right)^{n} e^{i h n(n-1) / 2}}{\lfloor n\rfloor !} e^{-i h n J_{3}}\left(J_{+}\right)^{n} \otimes e^{i h n J_{3}}\left(J_{-}\right)^{n}\right),
\end{gathered}
$$

where we have introduced the group-theoretic states $|J, M\rangle,-J \leqq M \leqq J$; together with operators $J_{ \pm}, J_{3}$ such that:

$$
J_{ \pm}|J, M\rangle=\sqrt{\lfloor J \mp M\rfloor\lfloor J \pm M+1\rfloor}|J, M \pm 1\rangle \quad J_{3}|J, M\rangle=M|J, M\rangle .
$$

These operators satisfy the commutation relations

$$
\left[J_{+}, J_{-}\right]=\left\lfloor 2 J_{3}\right\rfloor, \quad\left[J_{3}, J_{ \pm}\right]= \pm J_{ \pm} .
$$

2) For $0<\sigma<\sigma^{\prime}<\pi$, the $\xi$ fields obey the exchange algebra

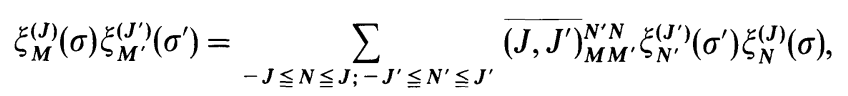




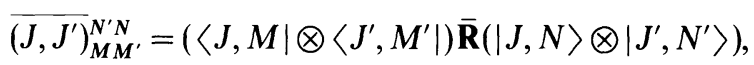

$$
\begin{aligned}
& \overline{\mathbf{R}}=e^{\left(2 i h J_{3} \otimes J_{3}\right)}\left(1+\sum_{n=1}^{\infty} \frac{\left(1-e^{-2 i h}\right)^{n} e^{-i h n(n-1) / 2}}{\lfloor n\rfloor !} e^{-i h n J_{3}}\left(J_{-}\right)^{n} \otimes e^{i h n J_{3}}\left(J_{+}\right)^{n}\right)
\end{aligned}
$$

3) The two exchange-formulae are related by the inverse-relation

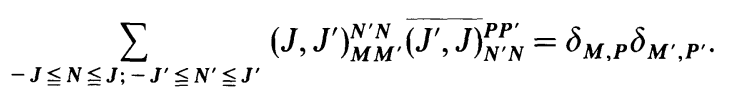

Going back to our problem, one sees that the above formulae give

$$
\begin{aligned}
e^{-\alpha-J_{1} \Phi(\sigma)} e^{-\alpha-J \Phi\left(\sigma^{\prime}\right)} \sim & \left(\frac{h}{16 \pi^{3}}\right)^{J_{1}+J} \sum_{M M^{\prime} P P^{\prime} N N^{\prime}} \xi_{M+P^{\prime}}^{\left(J_{1}+J\right)}(\sigma) \lambda\left(J_{1}, M ; J, P^{\prime}\right) \\
& \cdot A_{M, N}^{\left(J_{1}\right)} A_{M^{\prime}, N^{\prime}}^{(J)}\left(J_{1}, J\right)_{N M^{\prime}}^{P^{\prime} P} \lambda\left(J_{1}, P ; J, N^{\prime}\right) \xi_{N^{\prime}+P}^{\left(J_{1}+J\right)}(\bar{\sigma})
\end{aligned}
$$

It is sufficient for the present purpose to choose $J_{1}=1 / 2$. Then one has

$$
\begin{gathered}
(1 / 2, J)_{-1 / 2 M}^{M-1 / 2}=e^{i h M}, \quad(1 / 2, J)_{-1 / 2 M}^{M-11 / 2}=0, \quad(1 / 2, J)_{1 / 2 M}^{M 1 / 2}=e^{-i h M}, \\
(1 / 2, J)_{1 / 2 M}^{M+1-1 / 2}=e^{-i h / 2}\left(1-e^{2 i h}\right) \sqrt{\lfloor J-M\rfloor\lfloor J+M+1\rfloor}, \\
\lambda(1 / 2,-1 / 2 ; J, M)=\sqrt{\frac{\lfloor J-M+1\rfloor}{\lfloor 2 J+1\rfloor}} e^{i h / 2(J+M)}, \\
\lambda(1 / 2,1 / 2 ; J, M)=\sqrt{\frac{\lfloor J+M+1\rfloor}{\lfloor 2 J+1\rfloor}} e^{-i h / 2(J-M)} .
\end{gathered}
$$

The recursion for the $A$ coefficients reads

$$
\begin{aligned}
A_{P, N}^{(J+1 / 2)}= & e^{i h(P+1 / 4)}\left[\lambda(1 / 2,-1 / 2 ; J, P+1 / 2) A_{P+1 / 2, N+1 / 2}^{(J)} \lambda(1 / 2,-1 / 2 ; J, N+1 / 2)\right] \\
& +e^{-i h(P-1 / 4)}\left[\lambda(1 / 2,1 / 2 ; J, P-1 / 2) A_{P-1 / 2, N-1 / 2}^{(J)} \lambda(1 / 2,1 / 2 ; J, N-1 / 2)\right] \\
& +e^{-3 i h / 4}\left(1-e^{2 i h}\right) \sqrt{[J-P+3 / 2\rfloor\lfloor+P-1 / 2\rfloor} \\
& \cdot\left[\lambda(1 / 2,1 / 2 ; J, P-1 / 2) A_{P-3 / 2, N+1 / 2}^{(J)} \lambda(1 / 2,-1 / 2 ; J, N+1 / 2)\right] \\
& +e^{i h(P-1 / 4)} \kappa\left[\lambda(1 / 2,1 / 2 ; J, P-1 / 2) A_{P-1 / 2, N+1 / 2}^{(J)} \lambda(1 / 2,-1 / 2 ; J, N+1 / 2)\right] .
\end{aligned}
$$

For $J=1$ one finds the non-vanishing coefficients

$$
\begin{aligned}
A_{1,-1}^{(1)} & =e^{-i h}+\left(\kappa^{2}-1\right) e^{i h}, \quad A_{0,-1}^{(1)}=A_{1,0}^{(1)}=\sqrt{\lfloor 2\rfloor} \kappa \\
A_{1,1}^{(1)} & =A_{-1,-1}^{(1)}=e^{-i h}, \quad A_{0,0}^{(1)}=1 .
\end{aligned}
$$

This may be rewritten as

$$
A_{M, N}^{(1)}=\left\langle 1, M\left|e^{-i h J_{3}^{2}} \sum_{n=0}^{2} e^{i n n J_{3}} \frac{\left(J_{+}\right)^{n}}{\lfloor n\rfloor !} f_{n}\right| 1, N\right\rangle,
$$

where $f_{0}=1, f_{1}=\kappa, f_{2}=\kappa^{2}-1+e^{-2 i h}$. One is led to the general ansatz

$$
A_{M, N}^{(J)}=\langle J, M|A(\vec{J})| J, N\rangle ; \quad A(\vec{J}):=e^{-i h J_{3}^{2}} \sum_{n=0}^{\infty} e^{i h n J_{3}} \frac{\left(J_{+}\right)^{n}}{\lfloor n\rfloor !} f_{n} .
$$


It may be also verified to work for $J=3 / 2$ if $f_{3}=f_{1} f_{2}+f_{1}\left(e^{-4 i h}-1\right)$.

Let us turn to the general case. The above ansatz gives

$$
A_{M, N}^{(J)}=e^{-i h M N} \sqrt{\frac{\lfloor J-N\rfloor !\lfloor J+M\rfloor !}{\lfloor J+N\rfloor !\lfloor J-M\rfloor !}} \frac{f_{M-N}}{\lfloor M-N\rfloor !}
$$

for $M \geqq N,-J \leqq M \leqq J,-J \leqq N \leqq J$, and $A_{M, N}^{(J)}=0$ otherwise. Substitute into (3.18). After some computation, one sees that the ansatz solves the recurrence relation for the $A$ coefficients if the $f_{n}$ satisfy

$$
f_{n+1}=f_{n} f_{1}+f_{n-1}\left(e^{-2 i h n}-1\right) \text {. }
$$

In order to solve this last recursion, we define

where

$$
F_{n}(q):=\frac{f_{n}}{(q ; q)_{n}} \text { with } q=e^{-2 i n}
$$

$$
(y ; \rho)_{v}:=\prod_{r=0}^{\nu-1}\left(1-y \rho^{r}\right) .
$$

This last definition is standard in mathematics. Its relationship with the present conventions is expressed by the relation

$$
\lfloor a\rfloor_{v} \equiv \frac{\Gamma(a+v)}{\Gamma(a)}=(2 i \sin h)^{-v} e^{i h[v(v-1) / 2+v a]}\left(e^{-2 i h a} ; e^{-2 i h}\right)_{v} .
$$

Equation (3.23) becomes

$$
F_{n-2}-\kappa F_{n-1}+F_{n}=q^{n} F_{n}
$$

which is a $q$-analog of a recurrence formula for Hermite polynomials $[15,16]$ in the variable $\kappa / 2$. The solution is well known. Let $P(t, q):=\sum_{0}^{\infty} t^{n} F_{n}$, and $\kappa:=q^{a}+q^{-a}$.
One gets

so that

$$
\left(1-t q^{-a}\right)\left(1-t q^{a}\right) P(t, q)=P(q t, q)
$$

$$
P(t, q)=\prod_{0}^{\infty} \frac{1}{\left(1-t q^{n-a}\right)\left(1-t q^{n+a}\right)} .
$$

As a parenthetical remark, we note that Eq. (3.27) with $\kappa=2$, also appears in the pyramid stacking of squares [17]. The initial condition is different and the solution much more complicated, however. Using the standard identity

$$
\prod_{0}^{\infty} \frac{1}{\left(1-x q^{n}\right)}=\sum_{0}^{\infty} \frac{x^{r}}{(q ; q)_{r}}
$$

one finally obtains

$$
f_{n}=\sum_{r+s=n, r \geqq 0, s \geqq 0} q^{a(r-s)} q^{r s / 2}\left(\begin{array}{l}
n \\
r
\end{array}\right),
$$

so that $A(\vec{J})$ may be written as

$$
A(\vec{J}):=e^{-i h J_{3}^{2}} \sum_{r, s=0}^{\infty} e^{i h n J_{3}} \frac{\left(J_{+}\right)^{r+s} q^{a(r-s)} q^{r s / 2}}{\lfloor r\rfloor !\lfloor s\rfloor !} .
$$


This compact formula has a universal form which is somewhat similar to the expression of the universal $R$ matrix Eq. (3.8).

\section{Operator-Algebra of the Local Fields Involving One Quantum-Deformation Parameter}

Here we establish the operator-algebra of the fields $\exp \left(-\alpha_{-} J \Phi(\sigma)\right)$ constructed in the previous section. Starting from Eq. (3.2), and choosing $\sigma>\sigma^{\prime}$, for definiteness, we may write the product of two operators of the family as

$$
\begin{aligned}
e^{-\alpha-J_{1} \Phi(\sigma)} e^{-\alpha-J_{2} \Phi\left(\sigma^{\prime}\right)}= & \left(\frac{h}{16 \pi^{3}}\right)^{J_{1}+J_{2}} \sum_{M_{1}, M_{2} N_{1}, N_{2}} A_{M_{1}, N_{1}}^{\left(J_{1}\right)} A_{M_{2}, N_{2}}^{\left(J_{2}\right)} \\
& \cdot \xi_{M_{1}}^{\left(J_{1}\right)}(\sigma) \xi_{N_{1}}^{\left(J_{1}\right)}(\bar{\sigma}) \xi_{M_{2}}^{\left(J_{2}\right)}\left(\sigma^{\prime}\right) \xi_{N_{2}}^{\left(J_{2}\right)}\left(\bar{\sigma}^{\prime}\right),
\end{aligned}
$$

and, using Eqs. (3.6), (3.7),

$$
\begin{aligned}
e^{-\alpha-\Phi(\sigma)} e^{-\alpha-J_{2} \Phi\left(\sigma^{\prime}\right)}= & \left(\frac{h}{16 \pi^{3}}\right)^{J_{1}+J_{2}} \sum_{M_{1}, M_{2} N_{1}, N_{2}} \sum_{M, N} A_{M_{1}, N}^{\left(J_{1}\right)} A_{M, N_{2}}^{\left(J_{2}\right)}\left(J_{1}, J_{2}\right)_{N M}^{M_{2} N_{1}} \\
& \cdot \xi_{M_{1}}^{\left(J_{1}\right)}(\sigma) \xi_{M_{2}}^{\left(J_{2}\right)}\left(\sigma^{\prime}\right) \xi_{N_{1}}^{\left(J_{1}\right)}(\bar{\sigma}) \xi_{N_{2}}^{\left(J_{2}\right)}\left(\bar{\sigma}^{\prime}\right) .
\end{aligned}
$$

It is convenient to write, making use of Eq. (3.8),

$$
\begin{aligned}
& A_{M_{1}, N}^{\left(J_{1}\right)} A_{M, N_{2}}^{\left(J_{2}\right)}\left(J_{1}, J_{2}\right)_{N M}^{M_{2} N_{1}}=\left\langle J_{1}, M_{1}|A(\vec{J})| J_{1}, N\right\rangle\left(\left\langle J_{1}, N\right| \otimes\left\langle J_{2}, M_{2}\right|\right) \\
& \cdot \tilde{R}\left(\left|J_{1}, N_{1}\right\rangle \otimes\left|J_{2}, M\right\rangle\right)\left\langle J_{2}, M|A(\vec{J})| J_{2}, N_{2}\right\rangle, \\
& \tilde{R} \equiv e^{\left(-2 i h J_{3} \otimes J_{3}\right)}\left(\sum_{n=0}^{\infty} \frac{\left(1-e^{2 i h}\right)^{n} e^{i h n(n-1) / 2}}{\lfloor n\rfloor !} e^{i h n J_{3}}\left(J_{+}\right)^{n} \otimes\left(J_{+}\right)^{n} e^{i h n J_{3}}\right) .
\end{aligned}
$$

$\tilde{R}$ is deduced from $R$ by taking the transposed in the second space. In operator-form, one gets

$$
\begin{aligned}
\sum_{M N} A_{M_{1}, N}^{\left(J_{1}\right)} A_{M, N_{2}}^{\left(J_{2}\right)}\left(J_{1}, J_{2}\right)_{N M}^{M_{2} N_{1}}= & \left(\left\langle J_{1}, M_{1}\right| \otimes\left\langle J_{2}, M_{2}\right|\right)[A(\vec{J}) \otimes 1] \tilde{R}[1 \otimes A(\vec{J})] \\
& \cdot\left(\left|J_{1}, N_{1}\right\rangle \otimes\left|J_{2}, N_{2}\right\rangle\right) .
\end{aligned}
$$

Our discussion will be based on the following two theorems.

Theorem (1). Introduce the co-product associated with $U_{q}(\operatorname{sl}(2))$ :

$$
\Lambda(J)_{ \pm}=J_{ \pm} \otimes e^{i h J_{3}}+e^{-i h J_{3}} \otimes J_{ \pm}, \quad \Lambda(J)_{3}=J_{3} \otimes 1+1 \otimes J_{3} .
$$

The operators $A$ obey the following co-multiplication law

$$
A(\vec{\Lambda}(J))=[A(\vec{J}) \otimes 1] \tilde{R}[1 \otimes A(\vec{J})]
$$

if the coefficients $f_{n}$ satisfy

$$
\frac{f_{\mu+v}}{\lfloor\mu\rfloor !\lfloor v\rfloor !}=\sum_{0 \leqq p \leqq \min (\mu, v)} \frac{f_{v-p}}{\lfloor v-p\rfloor !\lfloor\mu-p\rfloor !} \frac{f_{\mu-p}}{\lfloor p\rfloor !} \frac{\left(1-e^{2 i h}\right)^{p}}{\lfloor i h(p(p-1) / 2-p(\mu+v))} .
$$

Proof. Introduce

$$
x:=J_{+} \otimes e^{i h J_{3}}, \quad y:=e^{-i h J_{3}} \otimes J_{+} .
$$


They satisfy

$$
y x=x y e^{-2 i h} .
$$

It is a standard result, which may be derived by recursion, that the $q$-deformed binomial coefficients are really binomial coefficients for non-commuting variables of this type. Indeed, one has

$$
(x+y)^{N}=\sum_{r=0}^{N}\left(\begin{array}{c}
N \\
r
\end{array}\right) e^{-i h r(N-r)} x^{r} y^{N-r} .
$$

This allows to write the left-hand side of (4.7) in the form $\sum_{m n} x^{m} y^{n} a_{m n}$. Re-expressing the right-hand side in the same way and identifying term by term complete the derivation.

Theorem (2). Equation (4.8) holds if (3.23) is true and $f_{0}=1$.

Proof. First (4.8) coincides with (3.23) if $\mu=1$ or $v=1$. Thus the proof is equivalent to the statement that the quantity

$$
\begin{gathered}
X_{\mu, v}:=\sum_{0 \leqq p \leqq \min (\mu, v)} C_{\mu, v}^{p} f_{\mu-p} f_{v-p}, \\
C_{\mu, v}^{p}:=\left(\begin{array}{l}
p \\
\mu
\end{array}\right)\left(\begin{array}{l}
p \\
v
\end{array}\right)\left(1-e^{2 i h}\right)^{p}\lfloor p\rfloor ! e^{i h(p(p-1) / 2-p(\mu+v+1 / 2))}
\end{gathered}
$$

is only a function of the sum $\mu+v$. This fact is verified as follows. The $q$-binomial coefficients satisfy

Thus

$$
\left(\begin{array}{c}
m+1 \\
n
\end{array}\right)=e^{-i h n}\left(\begin{array}{c}
m \\
n
\end{array}\right)+e^{i h(s-n+1)}\left(\begin{array}{c}
m \\
n-1
\end{array}\right) .
$$

$$
\begin{aligned}
& C_{\mu, v+1}^{p}=e^{-2 i h p}\left[C_{\mu, v}^{p}+\beta_{\mu-p+1} e^{2 i h} C_{\mu, v}^{p-1}\right], \\
& C_{\mu+1, v}^{p}=e^{-2 i h p}\left[C_{\mu, v}^{p}+\beta_{\nu-p+1} e^{2 i h} C_{\mu, v}^{p-1}\right],
\end{aligned}
$$

where $\beta_{n}:=e^{-2 i n n}-1$ is the coefficient of $f_{n-1}$ in (3.23). Making use of (4.15) one finally deduces from (3.27) that indeed $X_{\mu, v}=X_{\mu+1, v-1}$ only depends upon the sum $\mu+v$.

Concerning the operator-algebra, it follows from Theorem (1) that Eq. (4.1) is equivalent to

$$
\begin{aligned}
e^{-\alpha-J_{1} \Phi(\sigma)} e^{-\alpha-J_{2} \Phi\left(\sigma^{\prime}\right)}= & \left(\frac{h}{16 \pi^{3}}\right)^{J_{1}+J_{2}} \sum_{M_{1}, M_{2} N_{1}, N_{2}} \xi_{M_{1}}^{\left(J_{1}\right)}(\sigma) \xi_{M_{2}}^{\left(J_{2}\right)}\left(\sigma^{\prime}\right) \\
& \cdot\left(\left\langleJ_{1}, M_{1}\left|\otimes\left\langle J_{2}, M_{2}\right|\right) A(\vec{\Lambda}(J))\left(\left|J_{1}, N_{1}\right\rangle \otimes\left|J_{2}, N_{2}\right\rangle\right)\right.\right. \\
& \cdot \xi_{N_{1}}^{\left(J_{1}\right)}(\bar{\sigma}) \xi_{N_{2}}^{\left(J_{2}\right)}\left(\bar{\sigma}^{\prime}\right) .
\end{aligned}
$$

The discussion of the fusion is based on the following fusion properties [12] of the $\xi$ fields:

$$
\begin{aligned}
\xi_{M_{1}}^{\left(J_{1}\right)}(\sigma) \xi_{M_{2}}^{\left(J_{2}\right)}\left(\sigma^{\prime}\right)= & \sum_{J=\left|J_{1}-J_{2}\right|}^{J_{1}+J_{2}} g_{J_{1}, J_{2}}^{J}\left(d\left(\sigma-\sigma^{\prime}\right)\right)^{\Delta(J)-\Delta\left(J_{1}\right)-\Delta\left(J_{2}\right)} \\
& \left.\cdot\left(J_{1}, M_{1} ; J_{2}, M_{2} \mid J\right)\left(\xi_{M_{1}+M_{2}}^{(J)}(\sigma)+\text { descendants }\right)\right\}
\end{aligned}
$$


where

$$
\Delta(J) \equiv-J(J+1) h / \pi-J .
$$

The $q$-Clebsch-Gordan coefficient $\left(J_{1}, M_{1} ; J_{2}, M_{2} \mid J_{1}, J_{2} ; J, M_{1}+M_{2}\right)$ is abbreviated by $\left(J_{1}, M_{1} ; J_{2}, M_{2} \mid J\right) . g_{J_{1}, J_{2}}^{J}$ is a number whose explicit form will not be needed $\left(g_{1 / 2, J}^{J-1 / 2}\right.$ is computed in Appendix A). Substituting (4.17) into (4.16) gives

$$
\begin{aligned}
e^{-\alpha-J_{1} \Phi(\sigma)} e^{-\alpha-J_{2} \Phi\left(\sigma^{\prime}\right)}= & \left(\frac{h}{16 \pi^{3}}\right)^{J_{1}+J_{1}} \sum_{M_{1}, M_{2} N_{1}, N_{2}} \sum_{J=\left|J_{1}-J_{2}\right|}^{J_{1}+J_{2}} \sum_{\bar{J}=\left|J_{1}-J_{2}\right|}^{J_{1}+J_{2}} g_{J_{1}, J_{2}}^{J} g_{J_{1}, J_{2}}^{J} \\
& \cdot\left\{\left(d\left(\sigma-\sigma^{\prime}\right)\right)^{\Delta(J)-\Delta\left(J_{1}\right)-\Delta\left(J_{2}\right)}\left(d\left(\sigma^{\prime}-\sigma\right)\right)^{\Delta(\bar{J})-\Delta\left(J_{1}\right)-\Delta\left(J_{2}\right)}\right. \\
& \cdot\left(J_{1}, M_{1} ; J_{2}, M_{2} \mid J\right)\left(\left\langle J_{1}, M_{1}\right| \otimes\left\langle J_{2}, M_{2}\right|\right) A(\vec{\Lambda}(J)) \\
& \cdot\left(\left|J_{1}, N_{1}\right\rangle \otimes\left|J_{2}, N_{2}\right\rangle\right)\left(J_{1}, N_{1} ; J_{2}, N_{2} \mid \bar{J}\right) \\
& \left.\cdot\left(\xi_{M_{1}+M_{2}}^{(J)}(\sigma)+\text { descendants }\right)\left(\xi_{N_{1}+N_{2}}^{(\bar{J})}(\bar{\sigma})+\text { descendants }\right)\right\} .
\end{aligned}
$$

Introduce the states

$$
\left|J, M, J_{1}, J_{2}\right\rangle=\sum_{M_{1}+M_{2}=M}\left(J_{1}, M_{1}, J_{2}, M_{2} \mid J\right)\left(\left|J_{1}, M_{1}\right\rangle \otimes\left|J_{2}, M_{2}\right\rangle\right) .
$$

Equation (4.18) can be rewritten as

$$
\begin{aligned}
e^{-\alpha_{-} J_{1} \Phi(\sigma)} e^{-\alpha_{-} J_{2} \Phi\left(\sigma^{\prime}\right)}= & \left(\frac{h}{16 \pi^{3}}\right)^{J_{1}+J_{2}} \sum_{M, N} \sum_{J=\left|J_{1}-J_{2}\right|}^{J_{1}+J_{2}} \sum_{J=\left|J_{1}-J_{2}\right|}^{J_{1}+J_{2}} g_{J_{1}, J_{2}}^{J} g_{J_{1}, J_{2}}^{J} \\
& \cdot\left\{\left(d\left(\sigma-\sigma^{\prime}\right)\right)^{\Delta(J)-\Delta\left(J_{1}\right)-\Delta\left(J_{2}\right)}\left(d\left(\sigma^{\prime}-\sigma\right)\right)^{\Delta(J)-\Delta\left(J_{1}\right)-\Delta\left(J_{2}\right)}\right. \\
& \cdot\left\langle J, M, J_{1}, J_{2}|A(\vec{\Lambda}(J))| \bar{J}, N, J_{1}, J_{2}\right\rangle\left(\xi_{M}^{(J)}(\sigma)+\text { descendants }\right) \\
& \left.\cdot\left(\xi_{N}^{(\bar{J})}(\bar{\sigma})+\text { descendants }\right)\right\} .
\end{aligned}
$$

Next, we make use of the recurrence relation for the C.G. coefficients:

$$
\begin{aligned}
\sqrt{\left\lfloor J \mp M_{1} \mp M_{2}\right\rfloor\left\lfloor J \pm M_{1} \pm M_{2}+1\right\rfloor} & \left(J_{1}, M_{1} ; J_{2}, M_{2} \mid J\right) \\
= & e^{i h M_{2}} \sqrt{\left\lfloor J_{1} \mp M_{1}\right\rfloor\left\lfloor \pm M_{1}+1\right\rfloor}\left(J_{1}, M_{1} \pm 1 ; J_{2}, M_{2} \mid J\right) \\
& +e^{-i h M_{1}} \sqrt{\left\lfloor J_{2} \mp M_{2}\right\rfloor\left\lfloor J_{2} \pm M_{2}+1\right\rfloor}\left(J_{1}, M_{1} ; J_{2}, M_{2} \pm 1 \mid J\right),
\end{aligned}
$$

which lead to

$$
\begin{aligned}
\Lambda\left(J_{ \pm}\right)\left|J, M, J_{1}, J_{2}\right\rangle & =\sqrt{\lfloor J \mp M\rfloor\lfloor J \pm M+1\rfloor}\left|J, M \pm 1, J_{1}, J_{2}\right\rangle, \\
\Lambda\left(J_{3}\right)\left|J, M, J_{1}, J_{2}\right\rangle & =M\left|J, M, J_{1}, J_{2}\right\rangle,
\end{aligned}
$$

as expected from the group-theoretical meaning of the C.G. coefficients. Moreover, the orthogonality relations of C.G. coefficients, that is

$$
\sum_{N_{1}+N_{2}=N}\left(J_{1}, N_{1} ; J_{2}, N_{2} \mid J\right)\left(J_{1}, N_{1} ; J_{2}, N_{2} \mid \bar{J}\right)=\delta_{J, \bar{J}}
$$

imply

$$
\left\langle J, M, J_{1}, J_{2} \mid \bar{J}, M^{\prime}, J_{1}, J_{2}\right\rangle=\delta_{J, \bar{J}} \delta_{M, M^{\prime}}
$$


Therefore

$$
\left\langle J, M, J_{1}, J_{2}|A(\vec{\Lambda}(J))| \bar{J}, N, J_{1}, J_{2}\right\rangle=\langle J, M|A(\vec{J})| J, N\rangle \delta_{J, \bar{J}},
$$

and thus the O.P.E. expansion reads

$$
\begin{aligned}
e^{-\alpha-J_{1} \Phi(\sigma)} e^{-\alpha-J_{2} \Phi\left(\sigma^{\prime}\right)}= & \sum_{J=\left|J_{1}-J_{2}\right|}^{J_{1}+J_{2}}\left(g_{J_{1}, J_{2}}^{J}\right)^{2}\left(\frac{h}{16 \pi^{3}}\right)^{J_{1}+J_{2}-J} \\
& \cdot\left|d\left(\sigma-\sigma^{\prime}\right)\right|^{2\left[\Delta(J)-\Delta\left(J_{1}\right)-\Delta\left(J_{2}\right)\right]}\left\{e^{-\alpha-J \Phi(\sigma)}+\text { descendants }\right\} .
\end{aligned}
$$

We terminate this section by checking the locality of the operators $e^{-\alpha-J \Phi(\sigma)}$. According to Eq. (4.7) we can also write

$$
\begin{aligned}
e^{-\alpha-J_{2} \Phi\left(\sigma^{\prime}\right)} e^{-\alpha-J_{1} \Phi(\sigma)}= & \left(\frac{h}{16 \pi^{3}}\right)^{J_{1}+J_{2}} \sum_{M_{1}, M_{2} N_{1}, N_{2}} \xi_{M_{2}}^{\left(J_{2}\right)}\left(\sigma^{\prime}\right) \xi_{M_{1}}^{\left(J_{1}\right)}(\sigma) \\
& \cdot\left(\left\langleJ_{2}, M_{1}\left|\otimes\left\langle J_{1}, M_{1}\right|\right) A(\vec{\Lambda}(J))\left(\left|J_{2}, N_{2}\right\rangle \otimes\left|J_{1}, N_{1}\right\rangle\right)\right.\right. \\
& \cdot \xi_{N_{2}}^{\left(J_{2}\right)}\left(\bar{\sigma}^{\prime}\right) \xi_{N_{1}}^{\left(J_{1}\right)}(\bar{\sigma}) .
\end{aligned}
$$

Indeed, $\bar{\sigma}^{\prime}-\sigma=\bar{\sigma}-\sigma^{\prime}$ is positive, so that the computation is the same as when we started from (4.1). Using the braiding-algebra Eqs. (3.6)-(3.13), one permutes the operators $\xi_{M_{2}}^{\left(J_{2}\right)}\left(\sigma^{\prime}\right), \xi_{M_{1}}^{\left(J_{1}\right)}(\sigma)$, and $\xi_{N_{2}}^{\left(J_{2}\right)}\left(\bar{\sigma}^{\prime}\right), \xi_{N_{1}}^{\left(J_{1}\right)}(\bar{\sigma})$, respectively. One makes use of the identity

$$
\bar{R}^{T}=R^{-1}
$$

to transform (4.27) into

$$
\begin{aligned}
e^{-\alpha-J_{2} \Phi\left(\sigma^{\prime}\right)} e^{-\alpha-J_{1} \Phi(\sigma)}= & \left(\frac{h}{16 \pi^{3}}\right)^{J_{1}+J_{2}} \sum_{M_{1}, M_{2} N_{1}, N_{2}} \xi_{M_{1}}^{\left(J_{1}\right)}(\sigma) \xi_{M_{2}}^{\left(J_{2}\right)}\left(\sigma^{\prime}\right) \\
& \cdot\left(\left\langle J_{2}, M_{2}\right| \otimes\left\langle J_{1}, M_{1}\right|\right) R^{-1} A(\vec{\Lambda}(J)) \\
& \cdot R\left(\left|J_{2}, N_{2}\right\rangle \otimes\left|J_{1}, N_{1}\right\rangle\right) \xi_{N_{1}}^{\left(J_{1}\right)}(\bar{\sigma}) \xi_{N_{2}}^{\left(J_{2}\right)}\left(\bar{\sigma}^{\prime}\right)
\end{aligned}
$$

Equation (4.28) is easily deduced from (3.14), if one expresses it in terms of $\tilde{R}$, and notices that this last matrix is invariant by exchange of the two spaces in the tensor-product. The basic quantum-group property of the $R$ matrix is to satisfy the relation $R \bar{\Lambda}=\Lambda R$, where $\bar{\Lambda}$ is the other co-product

$$
\bar{\Lambda}(J)_{ \pm}=J_{ \pm} \otimes e^{-i h J_{3}}+e^{i h J_{3}} \otimes J_{ \pm}, \quad \bar{\Lambda}(J)_{3}=\Lambda(J)_{3} .
$$

Thus Eq. (4.29) may be simplified since $R^{-1} A(\vec{\Lambda}(J)) R=A(\overrightarrow{\bar{\Lambda}}(J))$. Finally, one makes use of the identity

$$
\begin{aligned}
& \left(\left\langleJ_{1}, M_{1}\left|\otimes\left\langle J_{2}, M_{2}\right|\right) A(\overrightarrow{\vec{\Lambda}}(J))\left(\left|J_{1}, N_{1}\right\rangle \otimes\left|J_{2}, N_{2}\right\rangle\right)\right.\right. \\
& \quad=\left(\left\langleJ_{2}, M_{2}\left|\otimes\left\langle J_{1}, M_{1}\right|\right) A(\vec{\Lambda}(J))\left(\left|J_{2}, N_{2}\right\rangle \otimes\left|J_{1}, N_{1}\right\rangle\right),\right.\right.
\end{aligned}
$$

and arrives at an expression which is identical to the right-hand side of (4.16). Thus we get locality:

$$
\left[e^{-\alpha-J_{1} \Phi(\sigma)}, e^{-\alpha-J_{2} \Phi\left(\sigma^{\prime}\right)}\right]=0
$$




\section{The Complete Operator-Algebra}

In this last part, we deal with the most general operator $\exp \left(-\left(\alpha_{-} J+\alpha_{+} \hat{J}\right) \Phi\right)$. First, we define these fields by fusion. They will be normalized so that

$$
e^{-\alpha-J \Phi(\sigma)} e^{-\alpha+\hat{J} \Phi\left(\sigma^{\prime}\right)} \sim\left(\left(d\left(\sigma-\sigma^{\prime}\right)\right)^{-2 J \hat{J} / \pi} e^{-(\alpha-J+\alpha+\hat{J}) \Phi(\sigma)},\right.
$$

where $\sim$ means that one takes the leading order in the limit $\sigma \rightarrow \sigma^{\prime}$. As before, the divergent factor will be omitted. The left-hand side is given by Eqs. (3.1), (3.22). The right-hand side is computed using the fusion and braiding properties derived in ref. 10,11 , for an arbitrary $\xi$-field and an arbitrary $\hat{\xi}$-field, that is, for $\sigma>\sigma^{\prime}$,

$$
\begin{gathered}
\xi_{M}^{(J)}(\sigma) \xi_{\hat{M}}^{(\hat{J})}\left(\sigma^{\prime}\right) \sim e^{i \pi(M \hat{J}-\hat{M} J)} \xi_{M, \hat{M}}^{(J, \hat{J}}(\sigma), \\
\xi_{M}^{(J)}(\sigma) \hat{\xi}_{\hat{M}}^{(\hat{J})}\left(\sigma^{\prime}\right)=e^{-2 i \pi J \hat{J}} e^{2 i \pi(M \hat{J}-\hat{M} J)} \hat{\xi}_{\hat{M}}^{(\hat{J})}\left(\sigma^{\prime}\right) \xi_{M}^{(J)}(\sigma) .
\end{gathered}
$$

Making use of these relations one obtains

$$
\begin{aligned}
e^{-(\alpha-J+\alpha+\hat{J}) \Phi(\sigma)}= & \left(\frac{h}{16 \pi^{3}}\right)^{J}\left(\frac{\hat{h}}{16 \pi^{3}}\right)^{\hat{J}} \sum_{M N \hat{M} \hat{N}} A_{M N}^{(J)} \hat{A}_{\hat{M} \hat{N}}^{(\hat{J})} e^{-2 i \pi J \hat{J}} e^{i \pi[(M-N) \hat{J}+(\hat{M}-\hat{N}) J]} \\
& \cdot \xi_{M, \hat{M}}^{(J, \hat{J})}(\sigma) \xi_{N, \hat{N}}^{(J, \hat{J})}(\bar{\sigma}) .
\end{aligned}
$$

One may check that one gets the same result if, instead of (5.1), one uses

$$
e^{-\alpha+\hat{J} \Phi(\sigma)} e^{-\alpha-J \Phi\left(\sigma^{\prime}\right)} \sim e^{-(\alpha-J+\alpha+\hat{J}) \Phi(\sigma)} .
$$

Next we verify that the operator so defined is again local. This immediately follows from its definition (5.1) (or, equivalently (5.5) if one admits that the ordering between fusion and braiding is irrelevant: it is then a consequence of the locality of $\exp \left[-\alpha_{-} J \Phi(\sigma)\right]$ and $\exp \left[-\alpha_{+} \hat{J} \Phi(\sigma)\right]$ separately, as was shown in the previous section; and from the fact that $\exp \left[-\alpha_{-} J \Phi(\sigma)\right]$ and $\exp \left[-\alpha_{+} \hat{J} \Phi(\sigma)\right]$ are mutually local, as one easily sees since their braiding matrix is trivial. We shall nevertheless carry out the proof, as a consistency check, omitting the details of the calculations.

Consider

$$
\begin{aligned}
e^{-\left(\alpha-J_{1}+\alpha+\hat{J}_{1}\right) \Phi(\sigma)} e^{-\left(\alpha-J_{2}+\alpha+\hat{J}_{2}\right) \Phi\left(\sigma^{\prime}\right)} & =e^{-2 i \pi\left(J_{1} \hat{J}_{1}+J_{2} \hat{J}_{2}\right)}\left(\frac{h}{16 \pi^{3}}\right)^{J_{1}+J_{2}}\left(\frac{\hat{h}}{16 \pi^{3}}\right)^{\hat{J}_{1}+\hat{J}_{2}} \\
& \cdot \sum_{M_{j} N_{j} \hat{M}_{j} \hat{N}_{j}, j=1,2} A_{M_{1} N_{1}}^{\left(J_{1}\right)} \hat{A}_{\hat{M}_{1} \hat{N}_{1}}^{\left(\hat{J}_{1}\right)} e^{i \pi\left[\left(M_{1}-N_{1}\right) \hat{J}_{1}+\left(\hat{M}_{1}-\hat{N}_{1}\right) J_{1}\right]} \xi_{M_{1}, \hat{M}_{1}}^{\left(J_{1}, \hat{J}_{1}\right)}(\sigma) \xi_{N_{1}, \hat{N}_{1}}^{\left(J_{1}, \hat{J}_{1}\right)}(\bar{\sigma}) \\
& \cdot A_{M_{2} N_{2}}^{\left(J_{2}\right)} \hat{A}_{\hat{M}_{2} \hat{N}_{2}}^{\left(\hat{J}_{2}\right)} e^{i \pi\left[\left(M_{2}-N_{2}\right) \hat{J}_{2}+\left(\hat{M}_{2}-\hat{N}_{2}\right) J_{2}\right]} \xi_{M_{2}, \hat{M}_{2}}^{\left(J_{2}, \hat{J}_{2}\right)}\left(\sigma^{\prime}\right) \xi_{N_{2}, \hat{N}_{2}}^{\left(J_{2}, \hat{J}_{2}\right)}\left(\bar{\sigma}^{\prime}\right) .
\end{aligned}
$$

Note that since $\pi>\sigma>\sigma^{\prime}>0$, we have $\pi>\bar{\sigma}^{\prime}>\bar{\sigma}>\sigma>\sigma^{\prime}>0$. Next commute $\xi_{N_{1}, \hat{N}_{1}}^{\left(J_{1}, \hat{J}_{1}\right)}(\bar{\sigma})$ and $\xi_{M_{2}, \hat{M}_{2}}^{\left(J_{2}, \hat{J}_{2}\right)}\left(\sigma^{\prime}\right)$, making use of the general braiding relation, valid for $\pi>\sigma>\sigma^{\prime}>0$

$$
\xi_{M_{1}, \hat{M}_{1}}^{\left(J_{1}, \hat{J}_{1}\right)}(\sigma) \xi_{M_{2}, \hat{M}_{2}}^{\left(J_{2}, \hat{J}_{2}\right)}\left(\sigma^{\prime}\right)=\sum_{N_{1}, N_{2}, \hat{N}_{1}, \hat{N}_{2}} \mathscr{R}_{M_{1}, \hat{M}_{1} ; M_{2}, \hat{M}_{2}}^{N_{2}, \hat{N}_{2} ; N_{1}, \hat{N}_{1}} \xi_{N_{2}, \hat{N}_{2}}^{\left(J_{2}, \hat{J}_{2}\right)}\left(\sigma^{\prime}\right) \xi_{N_{1}, \hat{N}_{1}}^{\left(J_{1}, \hat{J}_{1}\right)}(\sigma)
$$

where

$$
\begin{aligned}
\mathscr{R}_{M_{1}, \hat{M}_{1} ; M_{2}, \hat{M}_{2}}^{N_{2}, \hat{N}_{2} ; N_{1}, \hat{N}_{1}}= & \left.\left(J_{1}, J_{2}\right)_{M_{1} M_{2}}^{N_{2} N_{1}} \hat{(} \hat{J}_{1}, \hat{J}_{2}\right)_{\hat{M}_{1} \hat{N}_{1} \hat{N}_{1}}^{\hat{M}_{2}} \\
& \cdot e^{i \pi\left[J_{2}\left(\hat{N}_{1}+\hat{M}_{1}\right)+\hat{J}_{2}\left(N_{1}+M_{1}\right)-J_{1}\left(\hat{N}_{2}+\hat{M}_{2}\right)-\hat{J}_{1}\left(N_{2}+M_{2}\right)\right]} e^{-2 i \pi\left(J_{1} \hat{J}_{1}+J_{2} \hat{J}_{2}\right)}
\end{aligned}
$$


which was derived in ref. 10. One gets

$$
\begin{aligned}
& e^{-\left(\alpha-J_{1}+\alpha+\hat{J}_{1}\right) \Phi(\sigma)} e^{-\left(\alpha-J_{2}+\alpha+\hat{J}_{2}\right) \Phi\left(\sigma^{\prime}\right)} \\
& =\left(\frac{h}{16 \pi^{3}}\right)^{J_{1}+J_{2}}\left(\frac{\hat{h}}{16 \pi^{3}}\right)^{\hat{J}_{1}+\hat{J}_{2}} \sum_{M_{j} N_{j} \hat{M}_{j} \hat{N}_{j}, j=1,2, M_{2}^{\prime}, N_{1}^{\prime}, \hat{M}_{2}^{\prime}, \hat{N}_{1}^{\prime}} e^{i \pi \varphi_{1}} A_{M_{1} N_{1}}^{\left(J_{1}\right)} \\
& \cdot\left(J_{1}, J_{2}\right)_{N_{1} M_{2}}^{M_{2}^{\prime} N_{1}^{\prime}} \hat{A}_{\hat{M}_{1} \hat{N}_{1}}^{\left(\hat{J}_{1}\right)} \xi_{M_{1}, \hat{M}_{1}}^{\left(J_{1}, \hat{J}_{1}\right)}(\sigma) \xi_{M_{2}^{\prime}, \hat{M}_{2}^{\prime}}^{\left(J_{2}, \hat{J}_{2}\right)}\left(\sigma^{\prime}\right) \\
& \left.\cdot A_{M_{2} N_{2}}^{\left(J_{2}\right)} \hat{(J} \hat{J}_{1}, \hat{J}_{2}\right)_{\hat{N}_{1}, \hat{M}_{2}}^{\hat{M}_{2}^{\prime} \hat{N}_{1}^{\prime}} \hat{A}_{\hat{M}_{2} \hat{N}_{2}}^{\left(\hat{J}_{2}\right)} \xi_{N_{1}^{\prime}, \hat{N}_{1}^{\prime}}^{\left(J_{1}, \hat{\sigma}_{1}\right)}(\bar{\sigma}) \xi_{N_{2}, \hat{N}_{2}}^{\left(J_{2}, \hat{J}_{2}\right)}\left(\bar{\sigma}^{\prime}\right) .
\end{aligned}
$$

$\varphi_{1}$ is given by

$$
\begin{aligned}
\varphi_{1}= & J_{1}\left(\hat{M}_{1}-2 \hat{M}_{2}^{\prime}-\hat{N}_{1}^{\prime}\right)+J_{2}\left(\hat{M}_{2}^{\prime}+2 \hat{N}_{1}^{\prime}-\hat{N}_{2}\right) \\
& +\hat{J}_{1}\left(M_{1}-2 M_{2}^{\prime}-N_{1}^{\prime}\right)+\hat{J}_{2}\left(M_{2}^{\prime}+2 N_{1}^{\prime}-N_{2}\right) .
\end{aligned}
$$

It does not depend upon $N_{1}, M_{2}, \hat{N}_{1}$, and $\hat{M}_{2}$ so that, by making use of Eq. (4.7), each term of the summation may be written as

$$
\begin{gathered}
e^{i \pi \varphi_{1}}\left(\left\langleJ_{1}, M_{1}\left|\otimes\left\langle J_{2}, M_{2}^{\prime}\right|\right) A(\vec{\Lambda}(J))\left(\left|J_{1}, N_{1}^{\prime}\right\rangle \otimes\left|J_{2}, N_{2}\right\rangle\right)\right.\right. \\
\cdot\left(\hat{\langle} \hat{J}_{1}, \hat{M}_{1} \hat{\mid} \otimes \hat{\langle} \hat{J}_{2}, \hat{M}_{2}^{\prime} \hat{)}\right) \hat{A}(\overrightarrow{\bar{\Lambda}}(\hat{J}))\left(\hat{\mid} \hat{J}_{1}, \hat{N}_{1}^{\prime} \hat{\rangle} \otimes \hat{J} \hat{J}_{2}, \hat{N}_{2} \hat{\rangle}\right) \\
\cdot \xi_{M_{1}, \hat{M}_{1}}^{\left(J_{1}, \hat{J}_{1}\right)}(\sigma) \xi_{M_{2}, \hat{M}_{2}^{\prime}}^{\left(J_{2}, \hat{J}_{2}\right)}\left(\sigma^{\prime}\right) \xi_{N_{1}^{\prime}, \hat{N}_{1}^{\prime}}^{\left(J_{1}, \hat{J}_{1}\right)}(\bar{\sigma}) \xi_{N_{2}, \hat{J}_{2}}^{\left(J_{2}, \hat{J}_{2}\right)}\left(\bar{\sigma}^{\prime}\right) .
\end{gathered}
$$

There remains to commute $\xi_{M_{1}, \hat{M}_{1}}^{\left(J_{1}, \hat{J}_{1}\right)}(\sigma)$ with $\xi_{M_{2}^{\prime}, \hat{M}_{2}^{\prime}}^{\left(J_{2}, \hat{J}_{2}\right)}\left(\sigma^{\prime}\right)$ and $\xi_{N_{1}^{\prime}, \hat{N}_{1}^{\prime}}^{\left(J_{1}, \hat{J}_{1}\right)}(\bar{\sigma})$ with $\xi_{N_{2}, \hat{N}_{2}}^{\left(J_{2}, \hat{J}_{2}\right)}\left(\bar{\sigma}^{\prime}\right)$. Applying formulae (5.7), and (5.8), one sees that the calculation proceeds as in Sect. 4 for the hatted and unhatted braiding-matrices. After some algebra, the additional phase-factor is found to be precisely such that one obtains the same expression as in (5.6), but with the roles of 1,2 , and $\sigma, \sigma^{\prime}$ interchanged, respectively. Thus one concludes that one would have derived the same expression, if one had started from the product in reverse order; and locality follows.

The last point concerns closure by fusion. In the same way, as for locality one may consider that it is obvious: it follows from the discussion of Sect. 4 and from the definition (5.1), if one admits that fusion is associative. We nevertheless give the elements of the derivation for completeness. The general operator-product expansion of the $\xi_{M, \hat{M}}^{(J, \hat{J})}$ fields are given by

$$
\begin{array}{r}
\xi_{M_{1}, \hat{M}_{1}}^{\left(J_{1}, \hat{J}_{1}\right)}(\sigma) \xi_{M_{2}, \hat{M}_{2}}^{\left(J_{2}, \hat{J}_{2}\right)}\left(\sigma^{\prime}\right)=\sum_{J, \hat{J}=\left|J_{1}-J_{2}\right|}^{J_{1}+J_{2}}\left(\left(d\left(\sigma-\sigma^{\prime}\right)\right)^{p\left(J_{1}, J_{2}, \hat{J}_{1}, \hat{J}_{2} ; J, \hat{J}\right)} g_{J_{1} J_{2}}^{J} \hat{g}_{\hat{J}_{1} \hat{J}_{2}}^{\hat{J}_{2}}\right. \\
\cdot e^{i \pi\left[M_{1} \hat{J}_{2}+M_{2} \hat{J}_{1}-\hat{M}_{1} J_{2}-\hat{M}_{1} J_{2}\right]} e^{i \pi\left[\left(M_{1}+M_{2}\right)\left(\hat{J}-\hat{J}_{1}-\hat{J}_{2}\right)-\left(\hat{M}_{1}+\hat{M}_{2}\right)\left(J-J_{1}-J_{2}\right)\right]} \\
\left.\cdot\left(J_{1}, M_{1} ; J_{2}, M_{2} \mid J\right)\left(\hat{J}_{1}, \hat{M}_{1} ; \hat{J}_{2}, \hat{M}_{2} \hat{\mid} \hat{J}\right)\left(\xi_{M_{1}+M_{2}, \hat{M}_{1}+\hat{M}_{2}}^{(J, \hat{J})}(\sigma)+\cdots\right)\right),
\end{array}
$$

where $p\left(J_{1}, J_{2}, \hat{J}_{1} \hat{J}_{2} ; J, \widehat{J}\right):=\Delta_{\mathrm{Kac}}(J, \hat{J} ; C)-\Delta_{\mathrm{Kac}}\left(J_{1}, \hat{J}_{1} ; C\right)-\Delta_{\mathrm{Kac}}\left(J_{2}, \hat{J}_{2} ; C\right)$. Some general details about the structure of this formula are given at the end of the appendix. The quantities $g_{J_{1} J_{2}}^{J}$, and $\hat{g}_{\hat{J}_{1} \hat{J}_{2}}$ are coupling constants whose explicit expression is not needed for the proof, since they only depend upon the eigenvalues of the Casimir operators. This result was derived in ref. 12, apart from the second 
exponential on the second line. Since this factor is important for checking closure by fusion, we give some explanation of its origin. In ref. 12, the above general formula was derived by consistency with the co-product for the general fields $\xi_{M, \hat{M}}^{(J, \hat{J})}$. However, the formulae used for the latter (formula (2.53)) are not co-associative, namely, applying them twice to "add" three irreducible representations, one gets a result that depends upon the ordering chosen to combine them. We modify the general co-product in order to achieve co-associativity. For this formulae (2.53) of ref. 12 are to be replaced by

$$
\begin{aligned}
& J_{ \pm}^{(12)}=e^{\mp i \pi\left(\hat{J}^{(12)}-\hat{J}^{(1)}-\hat{J}^{(2)}\right)}\left(J_{ \pm}^{(1)} e^{i h J_{3}^{(2)} \mp i \pi \hat{J}^{(2)}}+e^{-i h J_{3}^{(1)} \mp i \pi \hat{J}^{(1)}} J_{ \pm}^{(2)}\right) \\
& \hat{J}_{ \pm}^{(12)}=e^{\mp i \pi\left(J^{(12)}-\hat{J}^{(1)}-J^{(2)}\right)}\left(\hat{J}_{ \pm}^{(1)} e^{i \hat{h} \hat{J}_{3}^{(2)} \mp i \pi J^{(2)}}+e^{-i h \hat{J}_{3}^{(1)} \mp i \pi J^{(1)}} \hat{J}_{ \pm}^{(2)}\right)
\end{aligned}
$$

where we used a self-explanatory notation that differs from the one of ref. 12. The additional factor in front includes the total spins $J^{(12)}$, and $\hat{J}^{(12)}$, which are given by the Casimir operators computed from the left-hand sides. This definition makes sense, since these Casimir operators are not sensitive to these factors. $J^{(12)}$, and $\widehat{J}^{(12)}$ of course commute with $J_{ \pm}^{(12)}, J_{3}^{(12)}, \widehat{J}_{ \pm}^{(12)}$, and $\widehat{J}_{3}^{(12)}$. It is easy to check that co-associativity holds, that is,

$$
J_{l}^{((12) 3)}=J_{l}^{(1(23))}, \quad \widehat{J}_{l}^{(12) 3)}=\widehat{J}_{l}^{(1(23))} .
$$

When the fusion-coefficients are derived, as in ref. 12 , by imposing its compatibility with the co-product, the new factor above gives the additional exponential in (5.12).

Finally, the general fusion-algebra is derived by applying (5.12) to each pair of terms appearing in (5.9), for $\sigma \rightarrow \sigma^{\prime}$ and $\bar{\sigma} \rightarrow \bar{\sigma}^{\prime}$, respectively. The calculation makes use of the basic identity (4.7) for the hatted and unhatted $A$ matrices. There are additional phase-factors which may be transformed to verify that the orthogonality of the q-C. G. coefficients (Eq. (4.24)) may be applied both for the hatted and unhatted ones. After some algebra, one gets the desired result:

$$
\begin{aligned}
& e^{-\left(\alpha-J_{1}+\alpha+\hat{J}_{1}\right) \Phi(\sigma)} e^{-\left(\alpha-J_{2}+\alpha+\hat{J}_{2}\right) \Phi\left(\sigma^{\prime}\right)} \\
& =\sum_{J, \hat{J}=\left|J_{1}-J_{2}\right|}^{J_{1}+J_{2}}\left(\frac{h}{16 \pi^{3}}\right)^{J_{1}+J_{2}-J}\left(\frac{\hat{h}}{16 \pi^{3}}\right)^{\hat{J}_{1}+\hat{J}_{2}-\hat{J}}\left|d\left(\sigma-\sigma^{\prime}\right)\right|^{2 p\left(J_{1}, J_{2}, \hat{J}_{1}, \hat{J}_{2} J, \hat{J}\right)} \\
& \quad \cdot\left(g_{J_{1} J_{2}}^{J}\right)^{2}\left(\hat{g}_{\hat{J}_{1} \hat{J}_{2}}^{\hat{J}}\right)^{2}\left\{e^{-(\alpha-J+\alpha+\hat{J}) \Phi(\sigma)}+\text { descendants }\right\} .
\end{aligned}
$$

\section{Outlook}

We have obtained a general construction of the operators $\exp \left(-\alpha_{-} J-\alpha_{+} \hat{J}\right) \Phi(\sigma)$ for arbitrary $J$ and $\hat{J}$ positive integers or half-integers. It is based on the introduction of the $A$ matrix which takes a universal form and has a close connection with the universal $R$ matrix and Clebsch-Gordan coefficients of the quantum group $U_{q}(\operatorname{sl}(2))$. This novel structure is related with the problem of factorizable scattering on the semi-line $[18,19]$. Moreover, locality of the inverse powers of the metric is equivalent to a relation involving four $R$ matrices and four $A$ matrices. A similar equation has just been introduced independently in ref. 20 for the $X X Z$-model with free ends. In this connection, we note an interesting 
generalisation of Eq. (4.16) namely,

$$
\begin{aligned}
\prod_{j=1}^{P} e^{-\alpha_{-} J_{j} \Phi\left(\sigma_{j}\right)}= & \left(\frac{h}{16 \pi^{3}}\right)^{\sum_{k}^{J_{k} J_{k}}} \sum_{M_{1}, N_{1} ; \cdots ; M_{P}, N_{P}}\left\{\left(\bigotimes_{j=1}^{P}\left\langle J_{j}, M_{j}\right|\right) A\left(J^{(1,2, \cdots, P)}\right)\right. \\
& \left.\cdot\left(\bigotimes_{k=1}^{P}\left|J_{k}, N_{k}\right\rangle\right) \prod_{r} \xi_{M_{r}}^{\left(J_{r}\right)}\left(\sigma_{r}\right) \prod_{l} \xi_{N_{s}}^{\left(J_{s}\right)}\left(\bar{\sigma}_{s}\right)\right\} .
\end{aligned}
$$

In this equation, $J^{(1,2, \cdots, P)}$ is a compact notation for the iterated co-product, which may be defined recursively by the equation

$$
J^{(1,2, \ldots, P)}=\Lambda\left(J^{(1)}, J^{(2, \ldots, P)}\right)
$$

where the notation is self explanatory, $\Lambda$ being the usual co-product (4.6). Due to the co-associativity, $J^{(1,2, \ldots, P)}$ satisfies,

$$
J^{(1,2, \ldots, P)}=\Lambda\left(J^{(1,2, \ldots, R),} J^{(R+1, \ldots, P)}\right), \quad \text { if } \quad 1 \leqq R \leqq P-1 .
$$

Equation (6.1) is derived by recurrence, repeating the proof carried out in Sect. 4, in order to establish Eq. (4.6). One of the standard properties of the $R$ matrix is equivalent to the identity

$$
\tilde{R}\left(J^{(1)}, J^{(3)}\right) \tilde{R}\left(J^{(2)}, J^{(3)}\right)=\tilde{R}\left(J^{(1,2)}, J^{(3)}\right) .
$$

This formula is easy to verify from the explicit expression (4.6), using the property indicated on Eqs. (4.9)-(4.11). Making use of (6.2), one deduces that

$$
\prod_{s=1, \ldots, P} \tilde{R}\left(J^{(s)}, J^{(P+1)}\right)=\tilde{R}\left(J^{(1, \ldots, P)}, J^{(P+1)}\right) .
$$

With this last relation, the recurrence proof is obvious. Formula (6.1) has an interesting structure. For instance, the decomposition of the general operatorproduct is reduced to the decomposition of co-products into irreducible representations, that is to the $q$-deformed Bethe ansatz. In general (6.1) illustrates the interplay between integrable algebraic structures and quantum gravity on the strip.

The future developments of the present study go in several directions. First, one should treat the case of rational theories, that is when $e^{i h}$ is a root of unity. For some special cases, it was already observed in ref. 4 that the Hilbert space of states has a remarkable truncation to a subset with positive highest-weights. In general, one can determine the set of critical exponents for gravity coupled to some world-sheet matter with boundaries. The factors $g_{J_{1} J_{2}}^{J}, \hat{g}_{\widehat{J}_{1} \hat{J}_{2}}^{\hat{J}_{2}}$ will then be needed to obtain the three-point functions. They may be determined, after some computation, following the recursive method of refs. 10, 12. Second one may consider strongly coupled gravity and try to derive a unitary truncation theorem similar to the one of refs. 11,12 . Third, one may use the $A$ matrix for studying integrable models on lattices with boundary. We hope to return to these problems in the future.

\section{Appendix A}

In this appendix, we derive the quantum-version of the Wronskian condition for the $\xi$ fields. This will also be useful to determine the normalisation of the classical 
$\xi$-functions by taking the classical limit. First recall some formulae of ref. 10. For this, it is convenient to adopt the slightly different notation of Appendix A of ref. 10: One writes $\psi^{\mu, v}$ instead of $\psi_{m}^{(J)}$ with $\mu=J-m$ and $v=J+m$ and introduces $V^{\mu, v}$ such that $V^{1,0}=V_{1}$ and $V^{0,1}=V_{2}$. In general (see formulae (A.14-A.18) of ref. 10), the $\psi$ 's and the $V$ 's are related by

$$
\psi^{\mu, v} \equiv\left(C^{\mu, v} D^{\mu, v}(\varpi)\right) V^{\mu, v} .
$$

The coefficients $C^{\mu, v}$ which do not depend upon $\varpi$ satisfy the relations

$$
\begin{gathered}
C^{1,0}=C^{0,1}=1, \\
C^{\mu+1, v}=C^{\mu, v} \frac{\Gamma_{0}(1+(\mu+v+1) h / \pi)}{\Gamma_{0}(1+(\mu+1) h / \pi)}, \\
C^{\mu, v+1}=C^{\mu, v} \frac{\Gamma_{0}(1+(\mu+v+1) h / \pi)}{\Gamma_{0}(1+(v+1) h / \pi)} .
\end{gathered}
$$

Since we want to adopt the same notations as in ref. 12, the ordinary gamma functions (not $q$-deformed) are denoted with an index zero since they correspond to a vanishing parameter $h$. Following ref. 12, it is the $q$-deformed gamma function with parameter $h$ which is denoted by $\Gamma(z)$. The dependence on $\varpi$ is contained in the coefficients $D^{\mu, v}(\varpi)$ which are determined by the recursion

$$
\begin{aligned}
& D^{\mu+1, v}(\varpi)=D^{\mu, v}(\varpi-1) \frac{\Gamma_{0}(-(\varpi+v) h / \pi)}{\left.\Gamma_{0}(-\varpi h / \pi)\right)} d_{1}(\varpi), \\
& D^{\mu, v+1}(\varpi)=D^{\mu, v}(\varpi+1) \frac{\Gamma_{0}((\varpi-\mu) h / \pi)}{\left.\Gamma_{0}(\varpi h / \pi)\right)} d_{2}(\varpi),
\end{aligned}
$$

with $D^{1,0} \equiv d_{1}$ and $D^{0,1} \equiv d_{2}$ satisfying

$$
d_{1}(\varpi) d_{2}(\varpi-1)=\Gamma_{0}(-\varpi h / \pi) \Gamma_{0}((\varpi-1) h / \pi) .
$$

One repeats the discussion of Appendix A of ref. 10 for the fusion of $V_{1,2}$ with $V^{\mu, \nu}$ keeping the two terms, following the proof of Theorem (2.8) of ref. 13. This leads to

$$
\begin{aligned}
V_{1} V^{\mu, v} \sim & \left(d\left(\sigma-\sigma^{\prime}\right)\right)^{-J h / \pi}\left(\frac{\Gamma_{0}(1+\varpi h / \pi) \Gamma_{0}(1+(\mu+v+1) h / \pi)}{\Gamma_{0}(1+(\varpi+v) h / \pi) \Gamma_{0}(1+(\mu+1) h / \pi)}\right) V^{\mu+1, v} \\
& +\left(d\left(\sigma-\sigma^{\prime}\right)\right)^{1+(J+1) h / \pi}\left(\frac{\Gamma_{0}(1+\varpi h / \pi) \Gamma_{0}(-1-(\mu+v+1) h / \pi)}{\Gamma_{0}(-2 v h / \pi) \Gamma_{0}((\varpi-\mu-1) h / \pi)}\right) V^{\mu, v-1} \\
V_{2} V^{\mu, v} \sim & \left(d\left(\sigma-\sigma^{\prime}\right)\right)^{-J h / \pi}\left(\frac{\Gamma_{0}(1-\varpi h / \pi) \Gamma_{0}(1+(\mu+v+1) h / \pi)}{\Gamma_{0}(1+(-\varpi+v) h / \pi) \Gamma_{0}(1+(v+1) h / \pi)}\right) V^{\mu, v+1} \\
& +\left(d\left(\sigma-\sigma^{\prime}\right)\right)^{1+(J+1) h / \pi}\left(\frac{\Gamma_{0}(1-\varpi h / \pi) \Gamma_{0}(-1-(\mu+v+1) h / \pi)}{\Gamma_{0}(-2 \mu h / \pi) \Gamma_{0}((-\varpi-v-1) h / \pi)}\right) V^{\mu, v-1}
\end{aligned}
$$

where $d\left(\sigma-\sigma^{\prime}\right)=1-e^{-i\left(\sigma-\sigma^{\prime}\right)}$. It is next straightforward to re-express the last two 
relations in terms of the $\psi$ fields using Eqs. (A.1)-(A.7). One gets

$$
\begin{gathered}
\psi_{ \pm 1 / 2}^{(1 / 2)} \psi_{m}^{(J)} \sim\left(d\left(\sigma-\sigma^{\prime}\right)\right)^{-J h / \pi} \frac{\lfloor\omega \mp J+m\rfloor}{\lfloor\omega\rfloor} \psi_{m \pm 1 / 2}^{(J+1 / 2)} \\
+\left(d\left(\sigma-\sigma^{\prime}\right)\right)^{1+(J+1) h / \pi} g_{J, 1 / 2}^{J-1 / 2} \frac{\lfloor\mp J+m\rfloor}{\lfloor\omega\rfloor} \psi_{m \pm 1 / 2}^{(J-1 / 2)}
\end{gathered}
$$

where

$$
g_{J, 1 / 2}^{J-1 / 2}=\Gamma_{0}(1+2 J h / \pi) \Gamma_{0}(-1-(2 J+1) h / \pi)
$$

is the coupling between spins $J, 1 / 2$ and $J-1 / 2$. The $\psi$ fields have been normalized in such a way that $g_{J, 1 / 2}^{J+1 / 2}=1$. Next we turn to the $\xi$ fields. For our purpose, we only need to extract the $J=0$ component of the operator-product of two $\xi^{(1 / 2)}$-fields. One has,

$$
\left.\left.\xi_{M}^{(1 / 2)}(\sigma) \xi_{M^{\prime}}^{(1 / 2)}\left(\sigma^{\prime}\right)=\sum_{m= \pm 1 / 2 m^{\prime}= \pm 1 / 2} \psi_{m}^{(1 / 2)}(\sigma) \mid J, \varpi-2 m\right)_{M}^{m} \mid J, \varpi\right)_{M^{\prime}}^{m^{\prime}} \psi_{m^{\prime}}^{(1 / 2)}\left(\sigma^{\prime}\right)
$$

and, according to Eq. (2.39) of ref. 12,

$$
\begin{aligned}
\sum_{M}= \pm 1 / 2 & (-1)^{1 / 2-M} e^{i h(1 / 2-M)} \xi_{-M}^{(1 / 2)}(\sigma) \xi_{M}^{(1 / 2)}\left(\sigma^{\prime}\right) \\
& =\sum_{m= \pm 1 / 2} \psi_{-m}^{(1 / 2)}(\sigma) C_{m}^{(1 / 2)}(\varpi) \psi_{m^{\prime}}^{(1 / 2)}\left(\sigma^{\prime}\right)
\end{aligned}
$$

where

$$
C_{ \pm 1 / 2}^{(1 / 2)}= \pm 2 i \sin (h) e^{i h / 2}\lfloor\varpi\rfloor
$$

Next, letting $\sigma \rightarrow \sigma^{\prime}$ and making use of (A.10), one gets

$$
\begin{aligned}
\sum_{M= \pm 1 / 2}(-1)^{1 / 2-M} e^{i h(1 / 2-M)} \xi_{-M}^{(1 / 2)}(\sigma) \xi_{M}^{(1 / 2)}\left(\sigma^{\prime}\right) \\
\\
\sim\left(d\left(\sigma-\sigma^{\prime}\right)\right)^{(1+3 h) / 2 \pi}\left(-2 i \pi e^{i h / 2}\right) \frac{\Gamma_{0}(1+h / \pi)}{\Gamma_{0}(2+2 h / \pi)}
\end{aligned}
$$

where the first term of (A.10) has disappeared, and where we used the fact that the second term involves $\psi_{0}^{(0)}$ which is a constant. Our conventions are such that it is equal to -1 . The first term of (A.10) is the spin-one contribution which disappears since the left member of (A.15) only involves the spin-zero contribution to the operator-product, according to the definition of ref. 12. Indeed, making use of the co-product (4.6), one may check that

$$
\begin{aligned}
& \sum_{M= \pm 1 / 2}(-1)^{1 / 2-M} e^{i h(1 / 2-M)} \Lambda(J)_{ \pm}\left[\xi_{-M}^{(1 / 2)}(\sigma) \xi_{M}^{(1 / 2)}\left(\sigma^{\prime}\right)\right] \\
& \equiv \sum_{M= \pm 1 / 2}(-1)^{1 / 2-M} e^{i h(1 / 2-M)}\left\{\sqrt{\lfloor J+M\rfloor\lfloor J-M+1\rfloor} e^{i h M} \xi_{-M+1}^{(1 / 2)}(\sigma) \xi_{M}^{(1 / 2)}\left(\sigma^{\prime}\right)\right. \\
& \left.\quad+\sqrt{\lfloor J-M\rfloor\lfloor J+M+1\rfloor} e^{i h M} \xi_{-M}^{(1 / 2)}(\sigma) \xi_{M+1}^{(1 / 2)}\left(\sigma^{\prime}\right)\right\}=0 .
\end{aligned}
$$

Equation (A.15) is the quantum-equivalent of the Wronskian condition for Schrödinger wave-functions. The classical Wronskian is regained as follows: Let 
$h \rightarrow 0$ keeping the product $h \varpi$ fixed. The quantum formulae have a smooth classical limit. Equation (A.15) becomes

$$
\xi_{-1 / 2}^{(1 / 2)}(\sigma) \xi_{1 / 2}^{(1 / 2)}\left(\sigma^{\prime}\right)-\xi_{1 / 2}^{(1 / 2)}(\sigma) \xi_{-1 / 2}^{(1 / 2)}\left(\sigma^{\prime}\right) \sim-2 \pi\left(\sigma-\sigma^{\prime}\right)
$$

and thus the Wronskian

$$
W\left[\xi_{1 / 2}^{(1 / 2)}, \xi_{-1 / 2}^{(1 / 2)}\right] \equiv \xi_{1 / 2}^{(1 / 2)}(\sigma) \frac{d}{d \sigma} \xi_{-1 / 2}^{(1 / 2)}(\sigma)-\xi_{-1 / 2}^{(1 / 2)}(\sigma) \frac{d}{d \sigma} \xi_{1 / 2}^{(1 / 2)}(\sigma)=2 \pi .
$$

Finally a last indication about our conventions may be useful. We work on the unit circle, and adopt the corresponding definition of primary fields. Our formulation is thus invariant by translation of $\sigma$, and not under translation of $z=e^{i \sigma}$ as is usual when one works on the complex plane. As a result the operatorproduct expansion of two primary fields $\mathscr{A}(\sigma)$ and $\mathscr{B}\left(\sigma^{\prime}\right)$ takes the form

$$
\mathscr{A}(\sigma) \mathscr{B}\left(\sigma^{\prime}\right)=\sum_{r}\left(d\left(\sigma-\sigma^{\prime}\right)\right)^{\Delta_{\vartheta_{r}}-\Delta_{\alpha}-\Delta_{*}}\left\{\mathscr{C}_{r}(\sigma)+\text { descendants }\right\},
$$

where $d\left(\sigma-\sigma^{\prime}\right)=1-e^{-i\left(\sigma-\sigma^{\prime}\right)}$. The $\Delta$ 's are the conformal weights, which for the fields $\xi_{M, \hat{M}}^{(J, \hat{J})}$ are given by Kac's formula:

$$
\Delta_{\mathrm{Kac}}(J, \hat{J} ; C)=\frac{C-1}{24}-\frac{1}{24}((J+\hat{J}+1) \sqrt{C-1}-(J-\hat{J}) \sqrt{C-25})^{2} .
$$

Acknowledgements. Part of this work was done while one of us (J.-L. G.) was visiting the Institute for Theoretical Physics at Santa Barbara. He benefited a great deal from the stimulating surrounding of the 1990 Workshop on Conformal Field Theory; and is greateful for the warm hospitality and generous financial support extended to him. This research was supported in part by the National Science Foundation under Grant No. PHY89-04035, supplemented by funds from the National Aeronautics and Space Administration, at the University of California at Santa Barbara.

\section{References}

1. Gervais, J.-L., Neveu, A.: Nucl. Phys. B199, 59 (1982)

2. Gervais, J.-L., Neveu, A.: Nucl. Phys. B202, 125 (1982)

3. Gervais, J.-L., Neveu, A.: Nucl. Phys. B224, 329 (1983)

4. Gervais, J.-L., Neveu, A.: Nucl. Phys. B238, 125 (1984); Nucl. Phys. B238, 396 (1984)

5. Gervais, J.-L., Neveu, A.: Nucl. Phys. B257 [FS14], 59 (1985)

6. Gervais, J.-L., Neveu, A.: Nucl. Phys. B264, 557 (1986)

7. Durhuus, B., Olesen, P., Petersen, J.: Nucl. Phys. B198, 157 (1982)

8. Durhuus, B., Nielsen, H., Olesen, P., Petersen, J.: Nucl. Phys. B196, 498 (1982)

9. Babelon, O.: Phys. Lett. B215, 253 (1988)

10. Gervais, J.-L.: Commun. Math. Phys. 130, 257 (1990)

11. Gervais, J.-L.: Phys. Lett. B243, 84 (1990)

12. Gervais, J.-L.: Commun. Math. Phys. 138, 301 (1991)

13. Gervais, J.-L.: On the algebraic structure of quantum gravity in two dimensions. ICTP preprint Trieste Conference on Topological Methods in Quantum Field Theories. Singapore: World Scientific

14. Bilal, A., Gervais, J.-L.: Nucl. Phys. B305 [FS23], 33 (1988); J. G. and Phys. Vol. 5 (2), 277 (1988) (Volume in honor of I.M. Gelfand 75th birthday)

15. Andrews, G.: q-series: Their development and application in analysis, number theory, 
combinatorics, physics, and computer algebra. Conference board of the Mathematical Sciences, Regional Conference in Mathematics, Vol. 66, AMS. (ed.)

16. Rodgers, L.: Second memoir on the expansion of certain infinite products. Proc. London Math Soc. 25, 318 (1984)

17. Privman, Svrakic,: J. Stat. Phys. 51, 1091 (1981)

18. Cherednik, I.: Theory. Math. Phys. 61, 977 (1984)

19. Sklyanin, E.: J. Phys. A21, 2375 (1988)

20. Kulish, P., Sklyanin, E.: Proceedings of the quantum group workshop, Euler Math. Inst. Leningrad. Berlin, Heidelberg, New York: Springer 1990

Communicated by K. Gawedzki 
